$$
\text { DOE/ET/35300--T2 }
$$

\title{
HTGR GENERIC TECHNOLOGY PROGRAM PLAN
}

(FY 80)

\section{Prepared by GENERAL ATOMIC COMPANY}

Contract No. DE-AM03-76SF00167 Program No. DE-AT03-76ET35300 


\section{DISCLAIMER}

This report was prepared as an account of work sponsored by an agency of the United States Government. Neither the United States Government nor any agency Thereof, nor any of their employees, makes any warranty, express or implied, or assumes any legal liability or responsibility for the accuracy, completeness, or usefulness of any information, apparatus, product, or process disclosed, or represents that its use would not infringe privately owned rights. Reference herein to any specific commercial product, process, or service by trade name, trademark, manufacturer, or otherwise does not necessarily constitute or imply its endorsement, recommendation, or favoring by the United States Government or any agency thereof. The views and opinions of authors expressed herein do not necessarily state or reflect those of the United States Government or any agency thereof. 


\section{DISCLAIMER}

Portions of this document may be illegible in electronic image products. Images are produced from the best available original document. 


\section{INTRODUCTION}

The program described in this document is a part of the overall program being undertaken by DOE, utilities and industry to allow realization of the national benefits to be derived from High Temperature Gas Cooled Reactor (HTGR) applications. This HTGR Generic Technology Program is a continuation of related research, development and demonstration (RD\&D) work which has been supported by DOE, utilities and General Atomic since the late 1950s.

The program includes all work currently foreseen by General Atomic as necessary to provide the base of design data, information and technology common to various HTGR reactor types in a time frame consistent with specific HTGR plant applications. The program includes design, development and analysis of HTGR generic reactor components and systems, design methods verification, testing, development and validation of design codes and assumptions, safety, reliability and availability analyses, fuel and fuel process development, basic materials design data development, studies of alternate designs and selected generic design improvements. All of these activities are coordinated with ongoing efforts on related programs at General Atomic, national labs and other national and international organizations and are managed through the HTGR Generic Technology Program management and support activities.

The program is planned towards providing the basic data common to the HTGR Steam Cycle (HTGR-SC) and HTGR Gas Turbine (HTGR-GT) or HTGR Process Heat (HTGR-PH) applications on a schedule consistent with the needs of each HTGR application.

The evaluation of a Pebble Bed core alternative to the reference prismatic core design will also be carried out under the Generic Technology Program.

This document provides an overall summary of the HTGR Generic Technology Program in FY-80. 
2. PURPOSE AND SCOPE

The purpose of the HTGR Generic Technology Program is to develop base technology, perform design and development common to the HTGR Steam Cycle, Gas Turbine and/or Process Heat Plants on a schedule consistent with specific applications.

The scope of the Generic Technology Program is shown diagrammatically as follows:

- The Generic Technology Program WBS

- The Base Technology WBS

- The Generic Component Design WBS

- The Generic Component DV\&S WBS

- Pebble Bed Study WBS

- The Technology Transfer WBS

- The Fresh Fuel Program WBS 


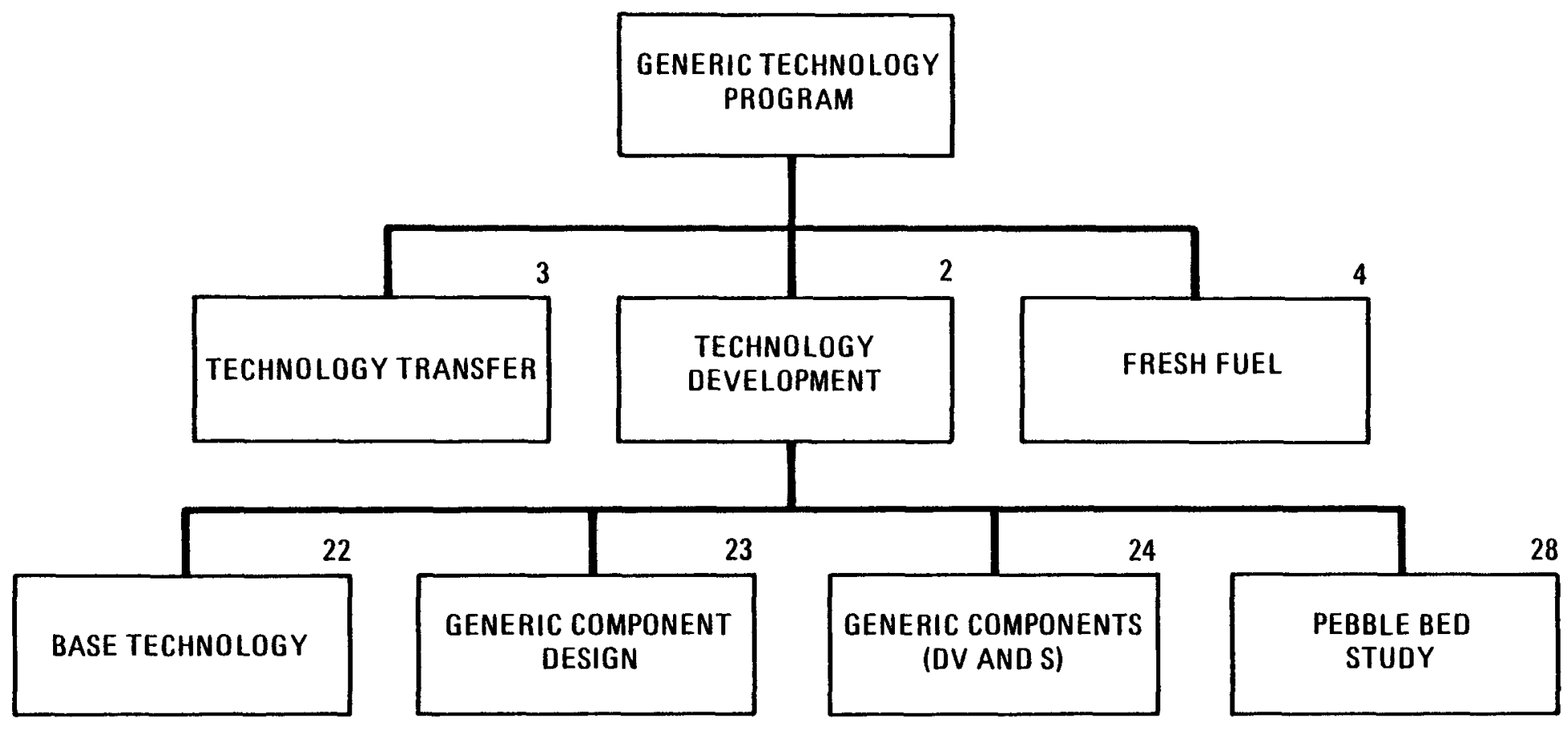

GA HTGR

GENERIC TECHNOLOGY PROGRAM WBS 


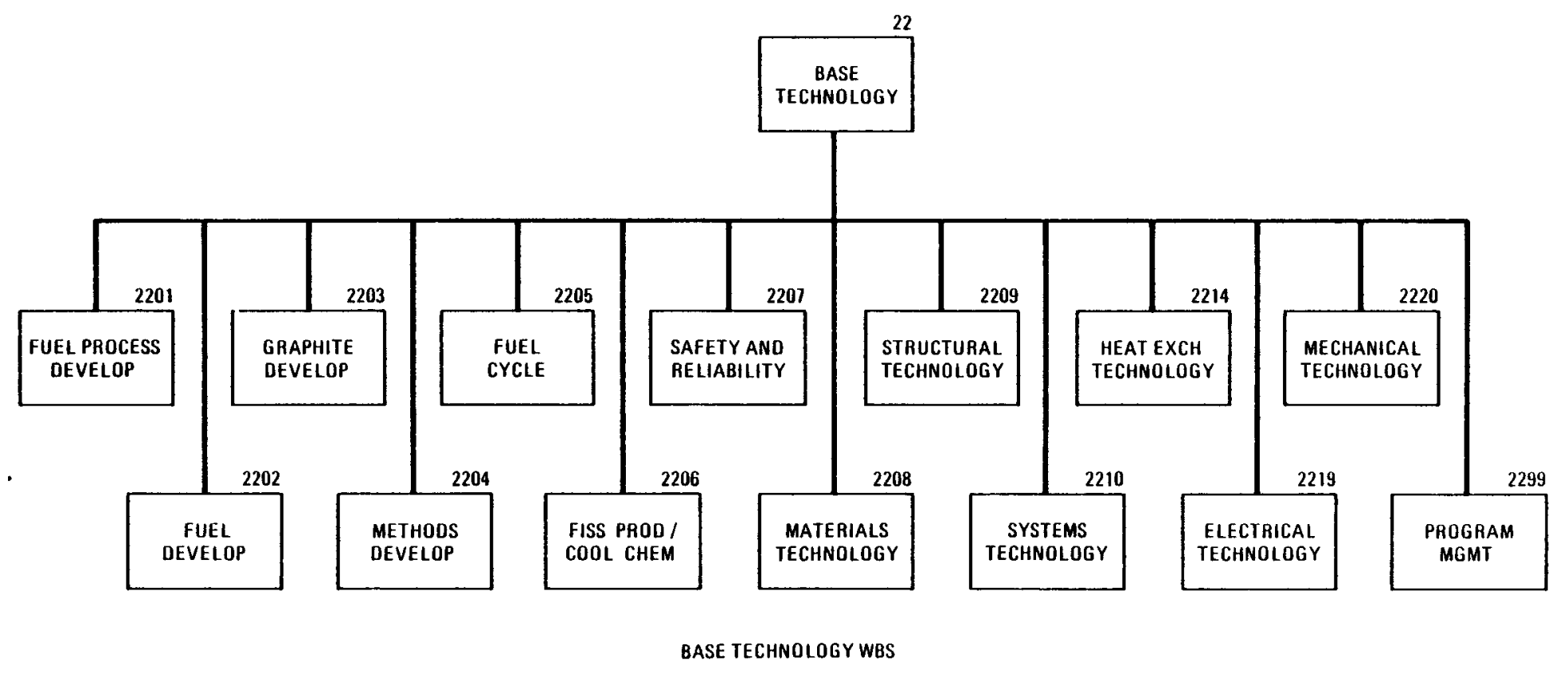




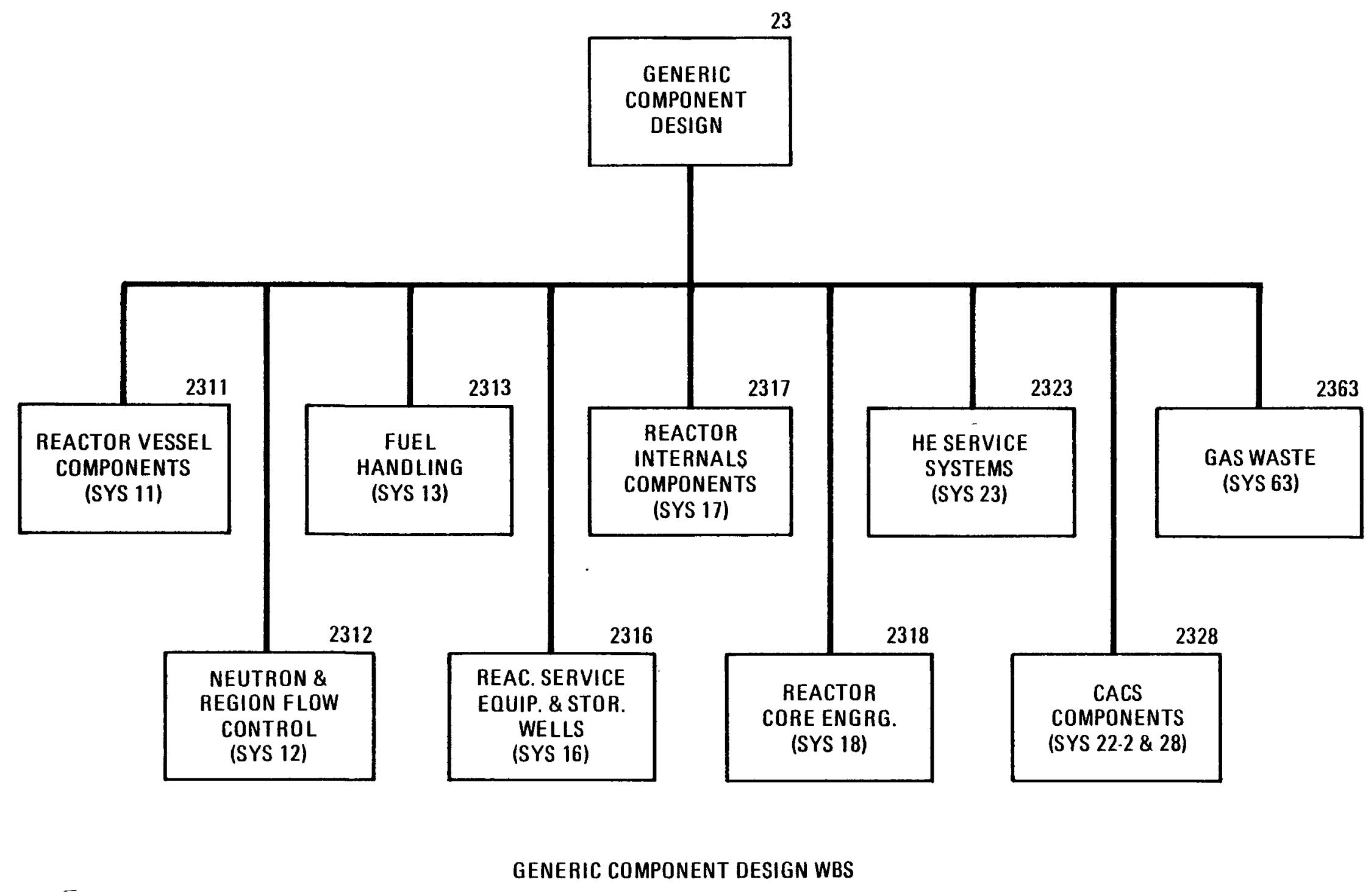




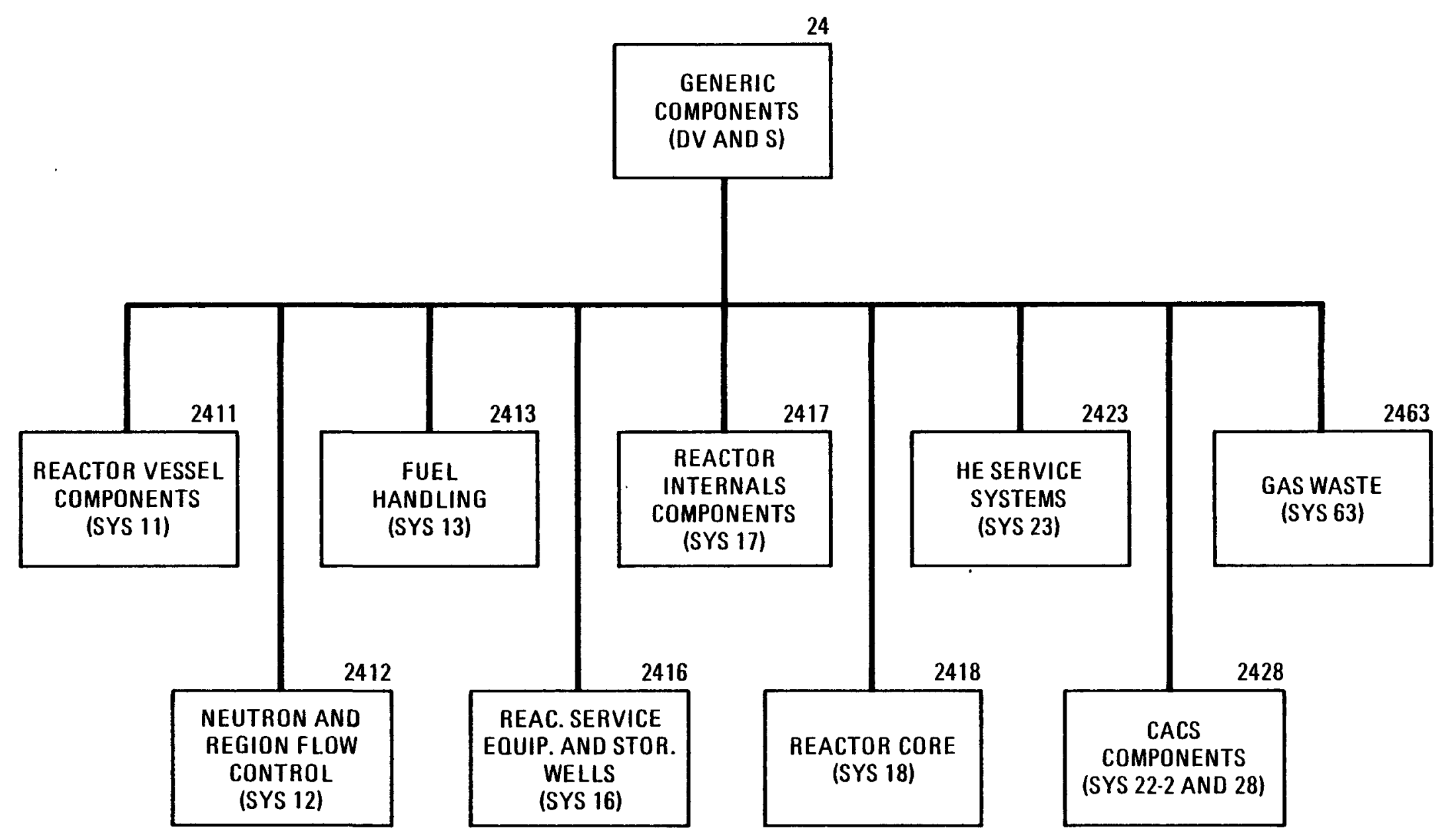

GENERIC COMPONENT DV AND S WBS 


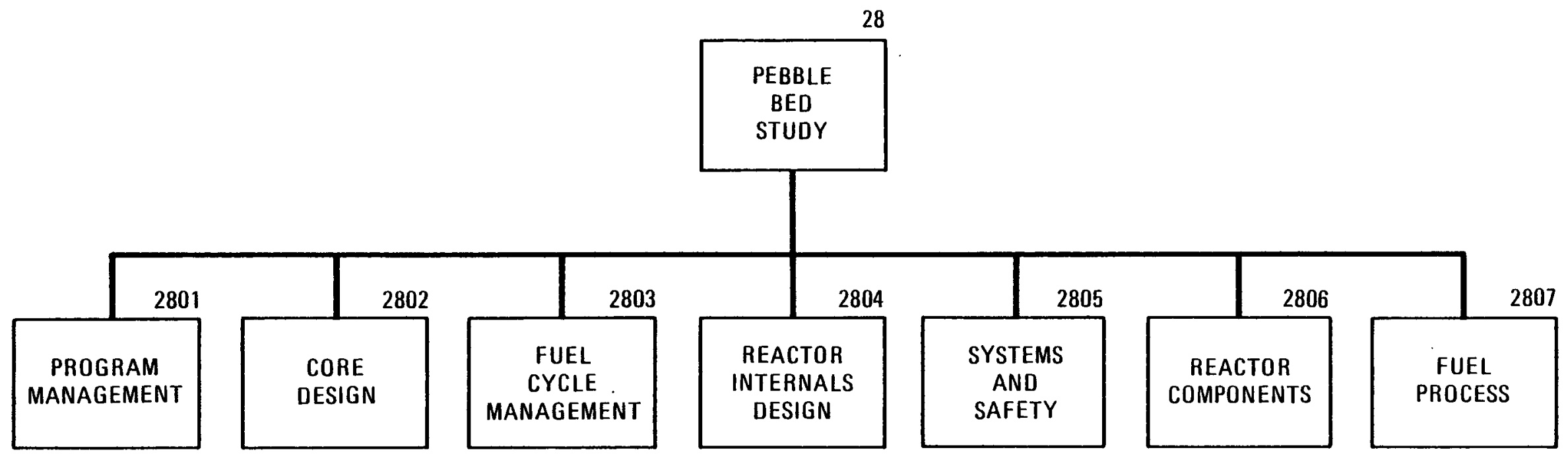

PEBBLE BED STUDY WBS 


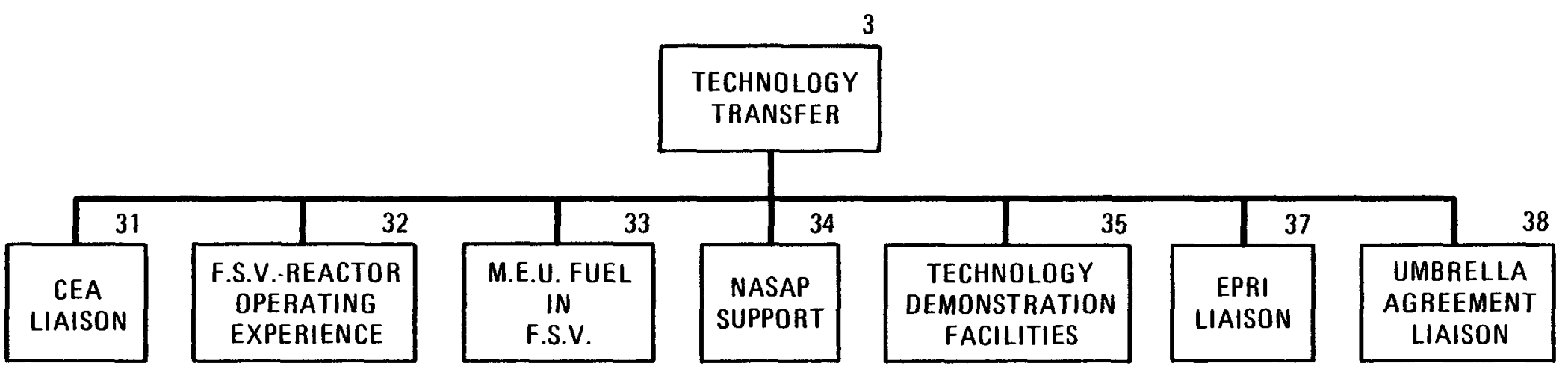

TECHNOLOGY TRANSFER WBS 


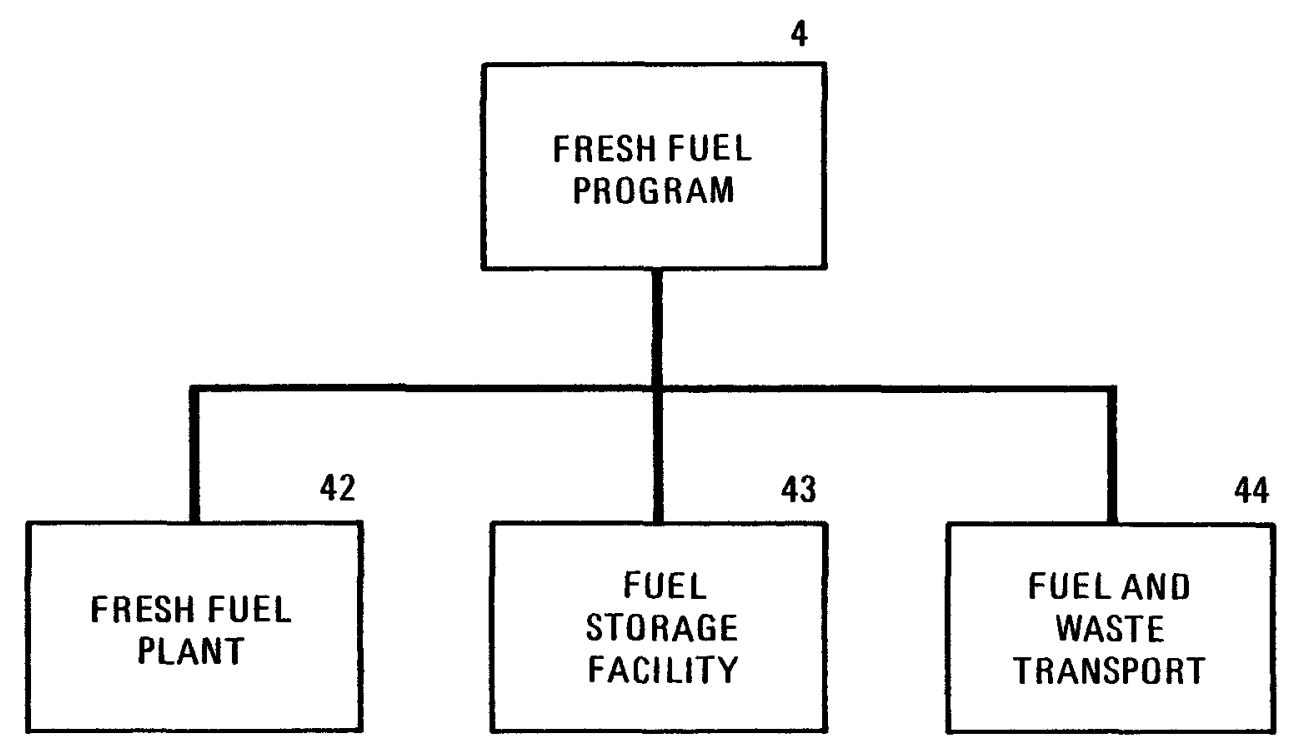

FRESH FUEL PROGRAM WBS 
3. MAJOR OBJECTIVES

The major objectives of the HTGR Generic Technology Program are as follows :

3.1 To develop and perform the design and analysis of generic HTGR components and systems, eliminating through redesign any deficiencies evident from Fort St. Vrain experience.

3.2 To resolve outstanding licensing and safety issues associated with HTR generic design technology and to perform safety and reliability analyses of HTGR generic components and systems.

3.3 To conduct a program of design verification tests to develop basic parameters and information for HTGR generic component designs and to verify the design methods and assumptions used.

3.4 To develop, update, validate and document HTGR generic design codes and associated assumptions.

3.5 To conduct programs of research and development to provide the necessary basic data, information and technology in the areas of structural materials, fuel materials, graphite, safety, fission products and coolant chemistry.

3.6 To perform studies of alternate design configurations and to continually improve the design bases of HTGR generic components and systems through selected product improvement programs.

3.7 To continually review the current base of national and international HTGR technology, together with the requirements of specific HTGR applications to ensure balanced and timely development of necessary generic design data and information. 
3.8 To provide an active and comprehensive program as a means for cooperation with interested foreign governments in the development of basic gas-cooled reactor technology.

3.9 To define, implement and utilize a management system that will ensure the orderly and efficient leadership, organization, planning and control of work towards all other aforementioned objectives. 
4. CONSTRAINTS AND ASSUMPTIONS

The major constraints and assumptions which apply to the HTGR Generic Technology Program as defined in this document are as follows:

4.1 "Generic" designs, data, information and technology developed under the HTGR Generic Technology Program must be common (or nearly so) to the HTGR-SC plant and either the HTGR-GT or HTGR-PH plants. Much of this generic information is also common to GCFR.

4.2 The plan as presented is consistent with GCRA's recommended National HTGR Program Plan and DOE's current financial guidance for $\mathrm{FY}-80$.

4.3 The HTGR Generic Technology Program is coordinated with the HTGR Base Technology Programs managed by ORNL and GEC.

4.4 The implications of any cooperative international government program on the programmatic or financial aspects of the program are included to the extent defined at the date of this report. The cooperative international program with the Federal Republic of Germany and other nations is expected to be further developed by DOE through the Umbrella Agreement.

4.5 The effect of recent changes in the French CEA Program have been included in the program plan, which now includes activities necessary to analyze and document all available data and to continue liaison on the remaining tests being performed on a subcontract from GA. 


\section{PRIORITIES AND SCHEDULE}

The HTGR Generic Program priorities are summarized below:

1. Tasks required to achieve HTGR Program definition and strategy selection, e.g.,

- Planning and Program development for advanced HTGRs

- Development of Program participants within U.S.

- Development of international cooperative programs

2. Application Studies

- Cogeneration-steam/electric

- Synfuel production from coal/lignite

- Synfuel production from oil shale

- Tar sands/heavy oil production

3. Tasks necessary to resolve critical technical and safety issues on advanced U.S. HTGRs, e.g.,

- High temperature materials development

- Core and internals design and development

- Thermal barrier (including hot duct) design and development

- Primary system environment - noise/flow distribution/ depressurization/oil or water ingress effects/fission product distribution

- Fuel and fuel process development

- Turbomachinery rotor, blade, and seal failure

- High temperature heat exchangers

- Primary system valves

4. Tasks required to significantly reduce GT/SC plant capital costs.

5. Ongoing commitments under International Agreements. 
6. Tasks to support the preapplication licensing program.

7. Special design studies to examine design alternatives, e.g.,

- IHX designs

- Replaceable thermal barrier

- Pebble bed core evaluation

- Turbine maintenance facility

- Alternate core/refueling system

- Enhanced safety study

8. Tasks that lead to the conceptual design defintion of advanced HTGRs .

The key program milestones are given in this section, a budget summary is given in Section 6.1 and the detailed program activities are given in Section 6.2, with the priorities listed above indicated for each activity.

The work currently covered by approved project work statement (PWS) under the Umbrella Agreement is separately identified in Section 6.2. Additional PWSs covering parts of the remaining work in Section 6.2 are on hold pending DOE direction on the overall Umbrella Agreement Implementation Plans.

\subsection{HTGR GENERIC TECHNOLOGY PROGRAM KEY MILESTONES}

Submit preapplication licensing LTRs to GCRA for transmittal to NRC

- General Design Criteria

$11 / 79$

- Time Dependent Release

$01 / 80$

- Accident Initiation and Progression

$06 / 80$

- Seismic Design Verification

$09 / 80$

Issue interim graphite design criteria 
Final report on design, construction and capabilities

Begin irradiation of $\mathrm{R} 2-\mathrm{K} 13$ fuel

Establish preliminary GT fuel specification

Prepare fuel cycle cost trend update

Establish fuel performance characteristics of a small

Complete analysis and documentation of closed out CEA tests

Prepare design guides based on FSV experience

Establish the reference thermal barrier materials for advanced HTGRs

Complete an assessment of the core temperature fluctuations as experienced at Fort St. Vrain and incorporate design changes if required on advanced HTGR's

Perform tests and provide design properties data 
6. PROGRAM SUMMARY FY -80

6.1 BUDGET SUMMARY

HTGR GENERIC TECHNOLOGY PROGRAM BUDGET

IN FY-80 ( $\left.\$ \times 10^{-3}\right)$

$22 x x$

2201

2202

2203

2204

2205

2206

2207

2208

2209

2210

2214

$23 \times X$

2312

2313

2316

2317

2318

2323

2328

$24 X X$

2411

2412

2418

$28 x x$

Pebble Bed Evaluation
852

1,812

1,144

550

453

766

361

2,028

570

122

254

8,912

17

119

5

219

1,505

3

335

2,203

311

11

485

807

518

518

3XXX Technology Transfer

$31 \times x$

$32 \mathrm{xx}$

$34 \mathrm{XX}$
CEA Liaison

FSV Reactor Operating Experience

NASAP Support
448

607

135 
4XXX Fresh Fuel Plant

XX99

Program Management, Licensing \& Support

1,665

Total Operating

1,665

Capital

15,315

425 
$2201 \quad$ Fuel Process

$\begin{array}{ll}\text { X-ray Plate Autoscanner } & 24 \\ \text { X-ray unit and facility } & 37 \\ \text { Spectrophotometer } & 10\end{array}$

2202 Fuel Development

Vacuum high temperature furnace $\quad 40$

2206 Fission Product \& Coolant Chemistry

Diffusion apparatus components 20

Precision lathe Technology 6

Metallographic polishing unit 6

Optical Microscope and Polaroid unit 5

2207 Safety and Reliability

High temperature furnace

2208 Material Technology

Environmental chamber $\quad 12$

Environmental chamber $\quad 16$

Test furnace 13

Hygrometer Study 1

Isolation table for sonic MOE 6

Diffusion hot welding press 56

Data analysis system 31

Test panel instrumentation $\quad 30$

Environmental chamber $\quad 19$

Gas furnace 26 
6.2 PROGRAM ACTIVITIES 


\begin{tabular}{|c|c|c|c|c|c|}
\hline $\begin{array}{l}\text { CWBS } \\
\text { TITLE }\end{array}$ & $\begin{array}{l}\text { CWBS } \\
\text { NO. }\end{array}$ & TASK AND DELIVERABLE & PRIORITY & $\begin{array}{l}\text { MILE- } \\
\text { STONE }\end{array}$ & $\begin{array}{l}\text { FY }-80 \\
\text { BUDGET } \$ 000 ' S\end{array}$ \\
\hline $\begin{array}{l}\text { Fresh Fuel } \\
\text { Process } \\
\text { Development } \\
(2201)\end{array}$ & $\begin{array}{l}22010105 \\
220103 \\
22010401 \\
22010403\end{array}$ & $\begin{array}{l}22 \text { BASE TECHNOLOGY PROGRAM } \\
\text { Fissile Kernel Process Development } \\
\text { Establish commercial feasibility of MEU } \\
\text { fissile kernel processes for advanced } \\
\text { HTGR fuel } \\
\text { O Issue report on GSP UC } 2 \\
\text { O Issue report on GSP UO } 2 \\
\text { HT-35 Driver Fuel Kernel Fabrication } \\
\text { Provide UCO driver fuel kernels to ORNL for } \\
\text { HT-35 and process development } \\
\text { o Ship fuel kernels to ORNL } \\
\text { Coating Process Development } \\
\text { Establish process development capability for } \\
\text { ZRC and Si-BISO coating } \\
\text { O Complete coater design study } \\
\text { Fuel Rod Process/Equipment Development } \\
\text { Design and fabricate wet sleeve mold and } \\
\text { assess 1ts performance characteristics } \\
\text { o Complete press design } \\
\text { Fuel Rod Curing Process Upgrade } \\
\text { Compare rod curing processes and identify } \\
\text { equipment for further development } \\
\text { o Issue recommendations } \\
\text { QC Techniques Development } \\
\text { Develop QC methods to meet preliminary MEU } \\
\text { specifications for advanced HTGR fuel } \\
\text { o Complete installation and shakedown of } \\
\text { equipment for improved coating thickness } \\
\text { and shape measurements }\end{array}$ & 3 & $1 / 80$ & 286 \\
\hline
\end{tabular}




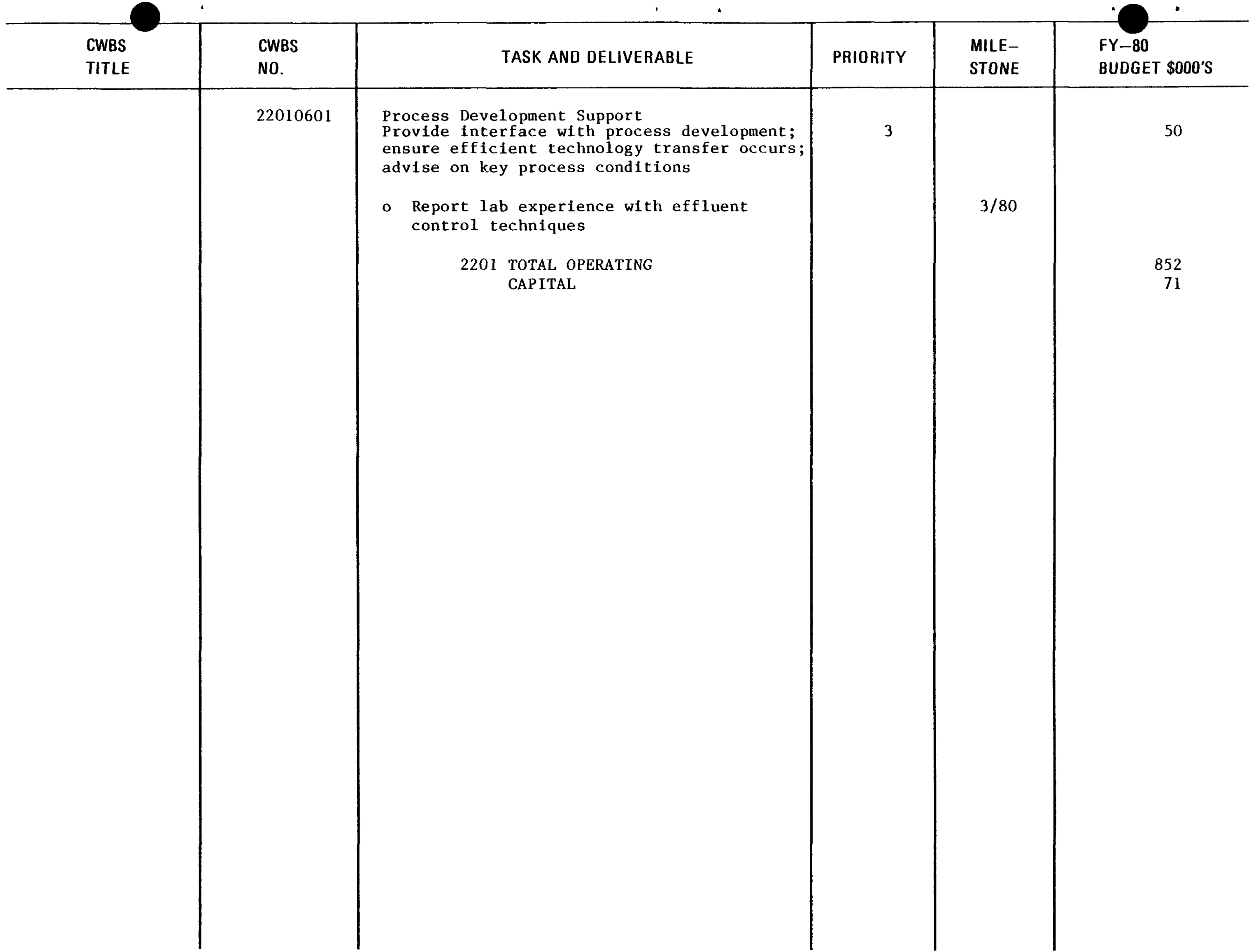




\begin{tabular}{|c|c|c|c|c|c|}
\hline $\begin{array}{l}\text { CWBS } \\
\text { TITLE }\end{array}$ & $\begin{array}{l}\text { CWBS } \\
\text { NO. }\end{array}$ & TASK AND DELIVERABLE & PRIORITY & $\begin{array}{l}\text { MILE- } \\
\text { STONE }\end{array}$ & $\begin{array}{l}\text { FY }-80 \\
\text { BUDGET } \$ 000 \text { 'S }\end{array}$ \\
\hline $\begin{array}{l}\text { Fuel } \\
\text { Devel opment } \\
(2202)\end{array}$ & $\begin{array}{l}22020101 \\
22020102 \\
22020103 \\
22020201\end{array}$ & $\begin{array}{l}\text { Accident Condition Test on Fuel Performance } \\
\text { Conduct isothermal post-irradiation heating } \\
\text { (PIH) experiments under accident conditions } \\
\text { irradiated samples of HEU and MEU fissile } \\
\text { and ThO } 2 \text { fertile fuel. Needed to develop } \\
\text { data base for fuel performance models } \\
\text { o Issue test plan, MEU scoping tests } \\
\text { o Complete } 4 \text { CHST's MEU fissile fuel } \\
\text { and issue data } \\
\text { Normal Condition Test on Fuel Performance } \\
\text { Perform thermal gradient PIH tests under } \\
\text { normal reactor conditions to develop data } \\
\text { base for fuel performance models and fission } \\
\text { product release predictions } \\
\text { o Issue test plan for HRB-15B MEU samples } \\
\text { o Report initial test results on HRB-15B } \\
\text { fuel } \\
\text { Fuel Performance Model Development } \\
\text { Develop fuel performance models to predict } \\
\text { expected levels of fission product release } \\
\text { under normal and hypothetical accident } \\
\text { conditions } \\
\text { o Complete LTR on time dependent release } \\
\text { o Complete LTR on results on CHST of TRISO } \\
\text { HEU UC } 2 \text { and Th0 } 2 \\
\text { Fuel Material Design and Specification } \\
\text { Develop fuel design specification and } \\
\text { pressure vessel models and maintain comput- } \\
\text { erized retrieval system } \\
\text { o Issue interim HTGR MEU fuel specifications }\end{array}$ & 3 & $\begin{array}{l}2 / 80 \\
9 / 80\end{array}$ & 200 \\
\hline
\end{tabular}




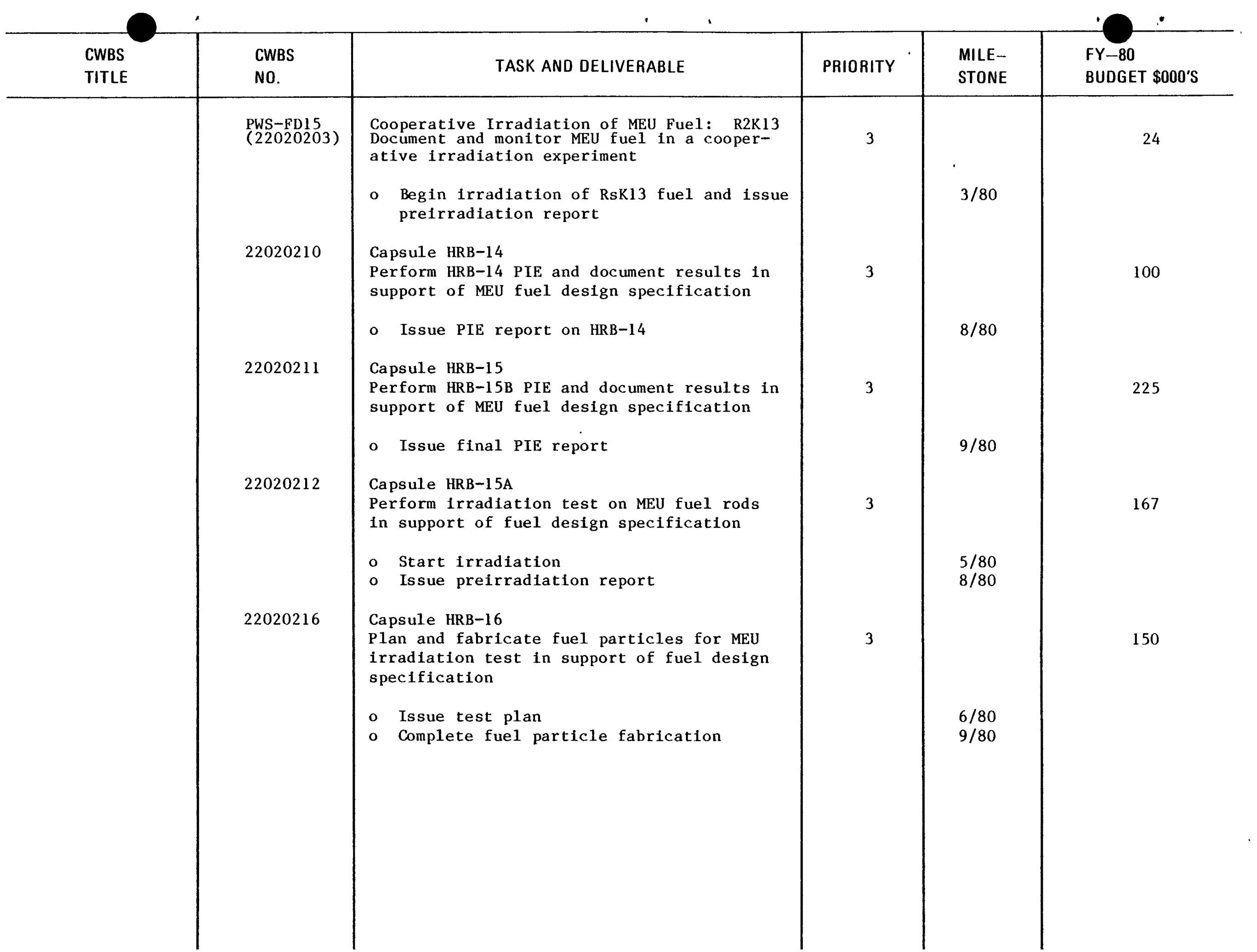




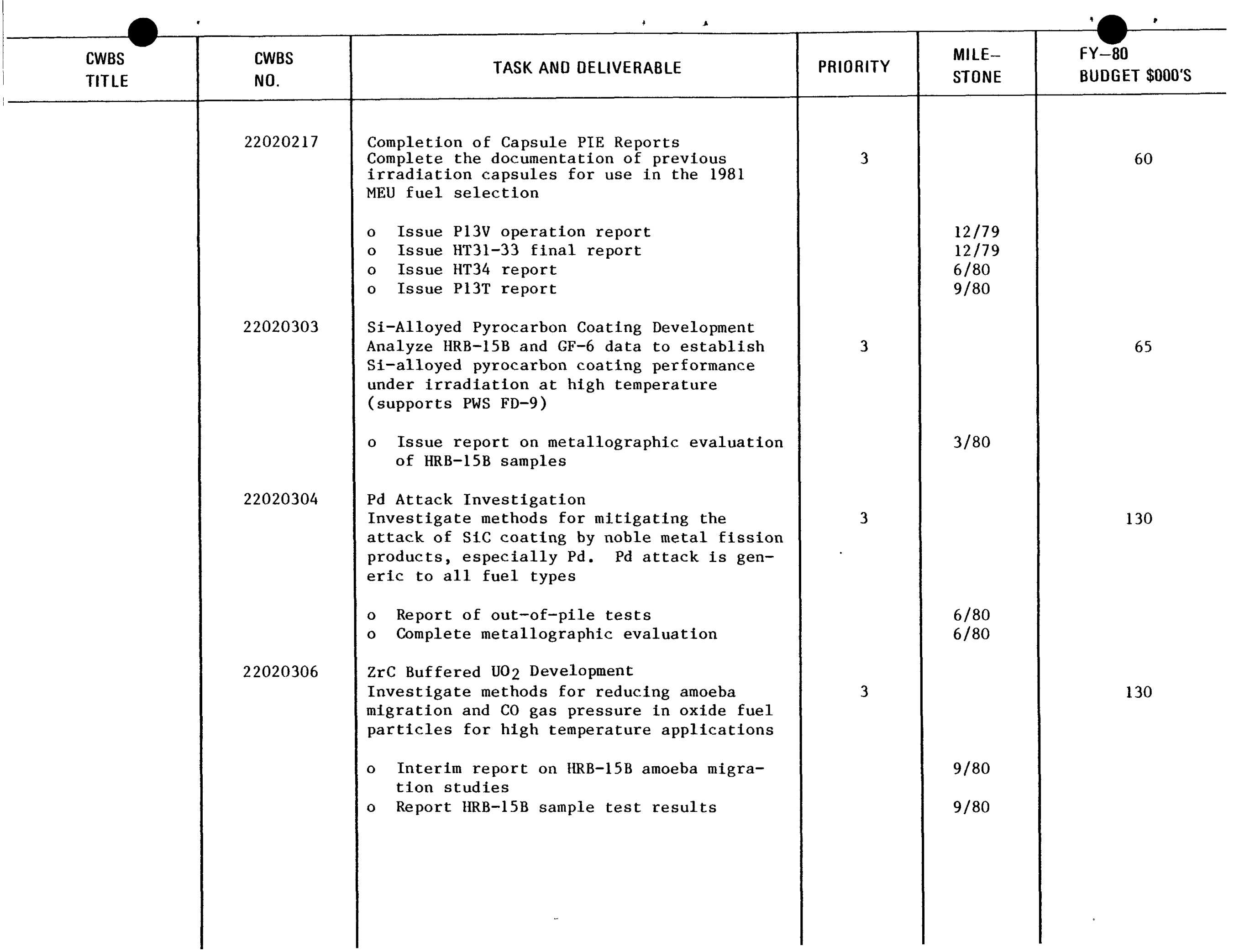




\begin{tabular}{|c|c|c|c|c|c|}
\hline $\begin{array}{l}\text { CWBS } \\
\text { TITLE }\end{array}$ & $\begin{array}{l}\text { CWBS } \\
\text { NO. }\end{array}$ & TASK AND DELIVERABLE & PRIORITY & $\begin{array}{l}\text { MILE- } \\
\text { STONE }\end{array}$ & $\begin{array}{l}\text { FY }-80 \\
\text { BUDGET } \$ 000 \text { 'S }\end{array}$ \\
\hline & $\begin{array}{l}22020310 \\
22020313 \\
22020406 \\
2202047\end{array}$ & $\begin{array}{l}\text { Improved Sic Coating Studies } \\
\text { Investigate the factors that influence SiC } \\
\text { microstructure and identify ways to improve } \\
\text { the strength and decrease fission metal dif- } \\
\text { fusion characteristics of this barrier layer } \\
\text { o Issue report on factors controlling micro- } \\
\text { structures and impurity distribution } \\
\text { o Complete evaluation of factors control- } \\
\text { ling strength } \\
\text { Coated Particle Stress Analysis Models } \\
\text { Develop analytical stress-analysis models to } \\
\text { predict fuel particle performance and to } \\
\text { compare model predictions with observed } \\
\text { irradiation behavior (supports FD-8) } \\
\text { o Issue report documenting TRISO model } \\
\text { Peach Bottom Fuel Test Elements } \\
\text { Finalize (review comments } \& \text { print) Peach } \\
\text { Bottom fuel test element documentation. } \\
\text { Provide support for preliminary safety anal- } \\
\text { ysis report on graphite and fuel performance } \\
\text { Including Pd attack data from FTE (supports } \\
\text { FD-14) } \\
\text { o Issue report } \\
\text { FSV Fuel Test Elements } 1 \text { - } 8 \\
\text { Prepare as-built drawings and complete } \\
\text { documentation of FTEs 1-8 in FSV reload 1. } \\
\text { Support LHTGR licensing and surveillance of } \\
\text { new candidate fuels in FSV } \\
\text { o Prepare pre-irradiation report } \\
\text { o Complete test plan for FTE-1 inspection } \\
\quad 2202 \text { ToTAL operATING } \\
\text { CAPITAL }\end{array}$ & 6 & $\begin{array}{c}3 / 80 \\
\\
. \\
5 / 80 \\
9 / 80\end{array}$ & $\begin{array}{r}1812 \\
40\end{array}$ \\
\hline
\end{tabular}




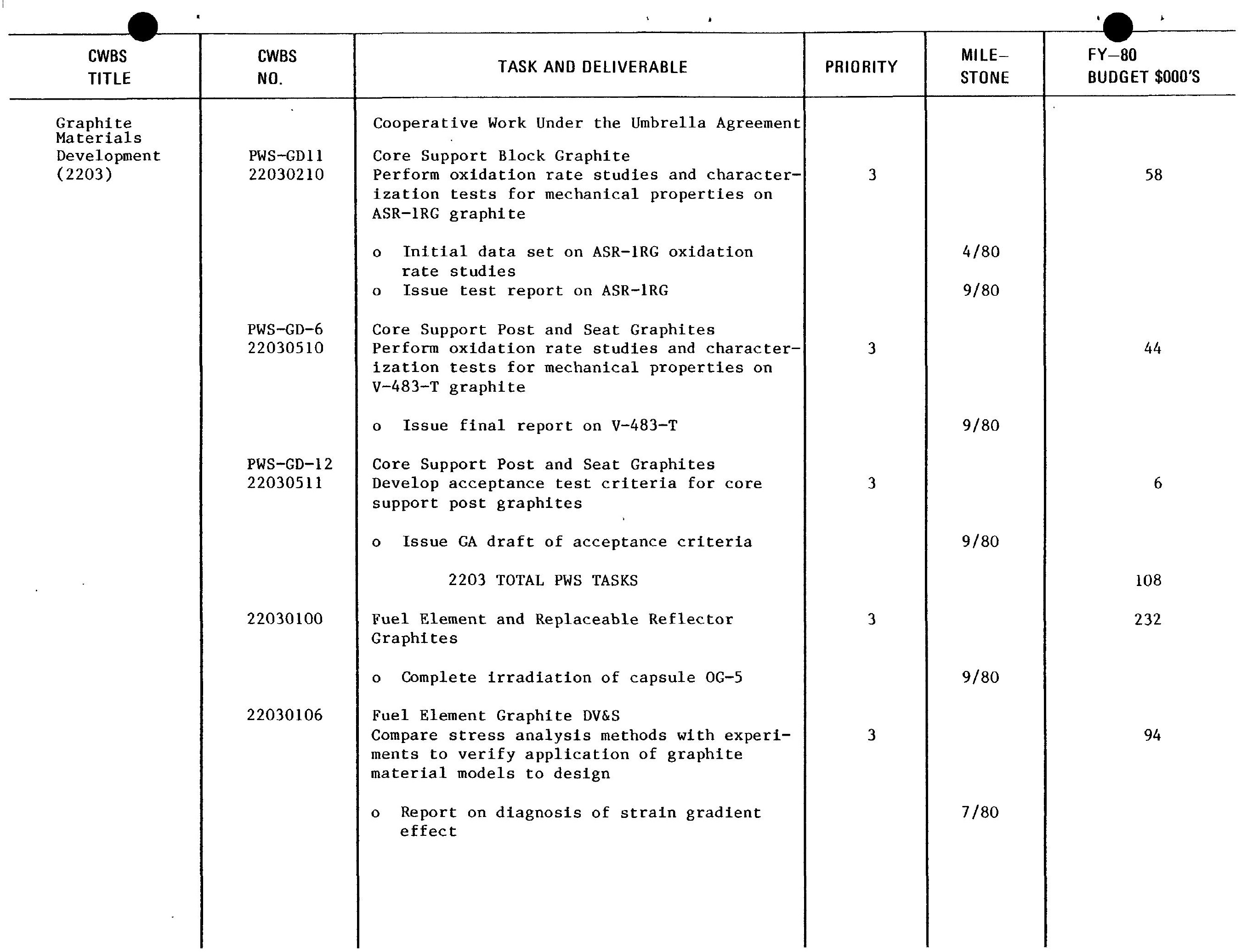




\begin{tabular}{|c|c|c|c|c|c|}
\hline $\begin{array}{l}\text { CWBS } \\
\text { TITLE }\end{array}$ & $\begin{array}{l}\text { CWBS } \\
\text { NO. }\end{array}$ & TASK AND DELIVERABLE & PRIORITY & $\begin{array}{l}\text { MILE- } \\
\text { STONE }\end{array}$ & $\begin{array}{l}\text { FY-80 } \\
\text { BUDGET } \$ 000 \text { 'S }\end{array}$ \\
\hline & $\begin{array}{l}22030200 \\
22030206 \\
22030500\end{array}$ & $\begin{array}{l}\text { Core Support Floor Block Graphites } \\
\text { Develop initial base of graphite material } \\
\text { properties for core support. block graphites } \\
\text { o Initial data set on TS-1621 oxidation } \\
\text { rate studies } \\
\text { Reactor Internals Graphite DV\&S } \\
\text { Provide graphite material modeling and spec- } \\
\text { ial design data required for core support } \\
\text { manent side reflector components design } \\
\text { verification } \\
\text { o Evaluation of internal damping tests } \\
\text { o Evaluation of thermal stress test } \\
\text { o Evaluation report of fracture mechanics } \\
\text { tests } \\
\text { o Final report on biaxial failure stress } \\
\text { test } \\
\text { Core Support Post and Seat Graphites } \\
\text { Develop initial base of graphite material } \\
\text { properties for core support post and seat } \\
\text { graphites } \\
\text { Issue data set for H451 instr. beam test } \\
\text { o } 2203 \text { ToTAL opERATING (including PWS) }\end{array}$ & 3 & $\begin{array}{l}12 / 79 \\
1 / 80 \\
8 / 80 \\
9 / 80\end{array}$ & 1144 \\
\hline
\end{tabular}




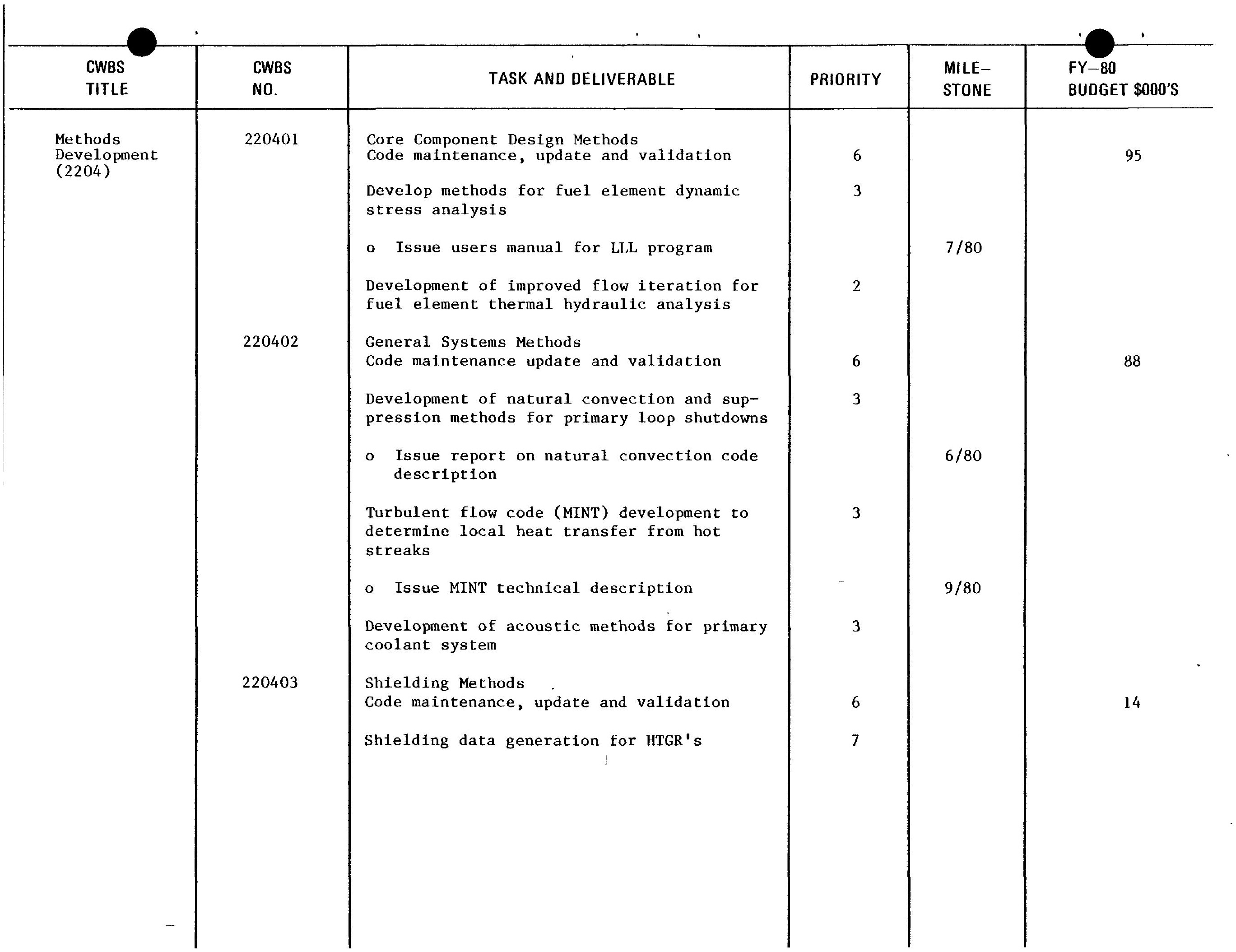




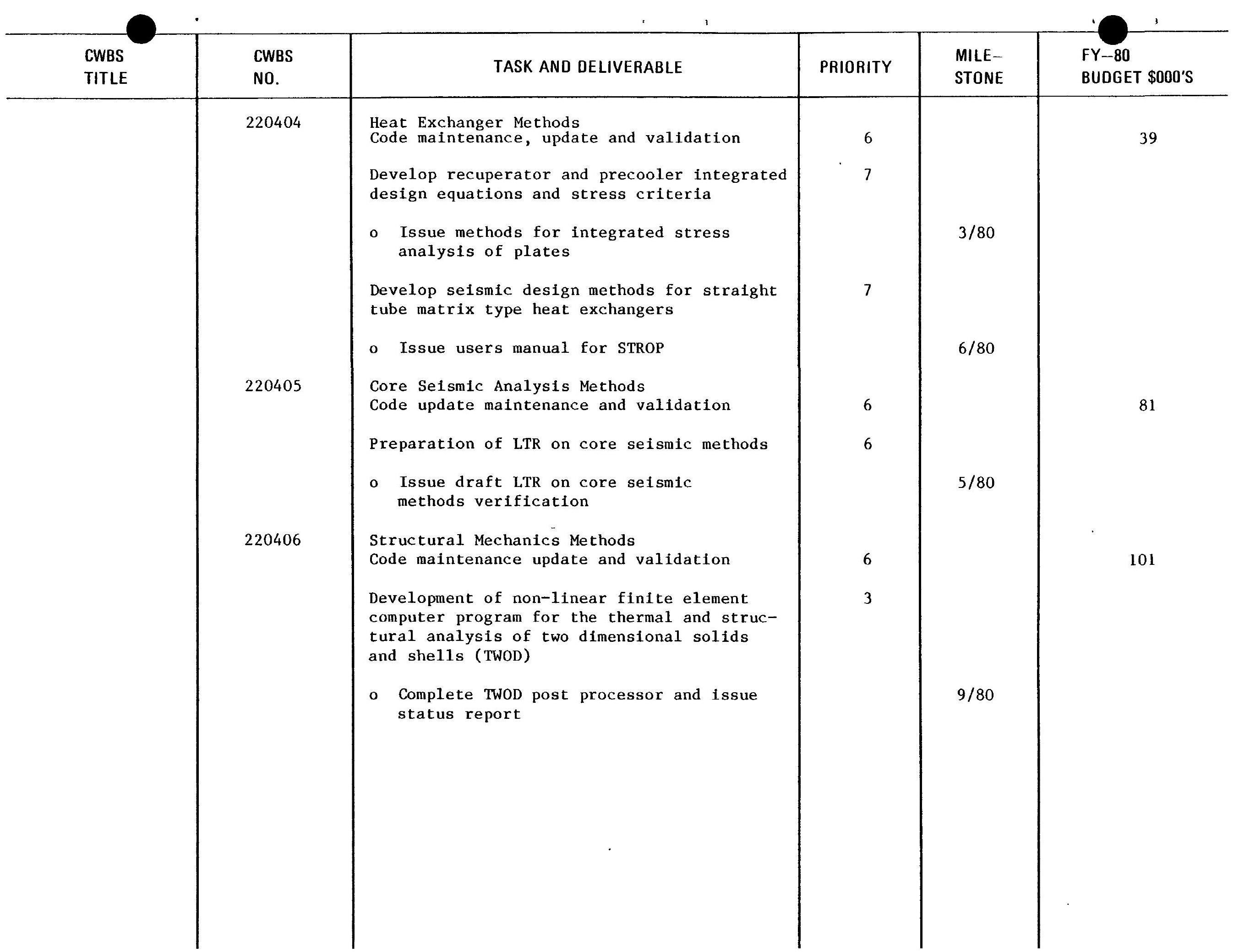




\begin{tabular}{|c|c|c|c|c|c|}
\hline $\begin{array}{l}\text { CWBS } \\
\text { TITLE }\end{array}$ & $\begin{array}{l}\text { CWBS } \\
\text { NO. }\end{array}$ & TASK AND DELIVERABLE & PRIORITY & $\begin{array}{l}\text { MILE- } \\
\text { STONE }\end{array}$ & $\begin{array}{l}\text { FY-80 } \\
\text { BUDGET } \$ 000 ' S\end{array}$ \\
\hline & $\begin{array}{l}220407 \\
220408\end{array}$ & $\begin{array}{l}\text { System Design Methods } \\
\text { Code maintenance, update and validation } \\
\text { o Complete COLUMN/PLENUM and issue } \\
\text { documentation } \\
\text { New code development } \\
\text { Upgrade natural convection code NATCIR } \\
\text { for enhanced core cooling studies } \\
\text { o Update NATCIR and issue documentation } \\
\text { Fuel Management Methods } \\
\text { Code maintenance update and verification } \\
\text { Statistical support for fuel particle } \\
\text { testing and design } \\
2204 \text { TOTAL OPERATING }\end{array}$ & $\begin{array}{l}6 \\
7\end{array}$ & $\begin{array}{c}9 / 80 \\
.\end{array}$ & 550 \\
\hline
\end{tabular}




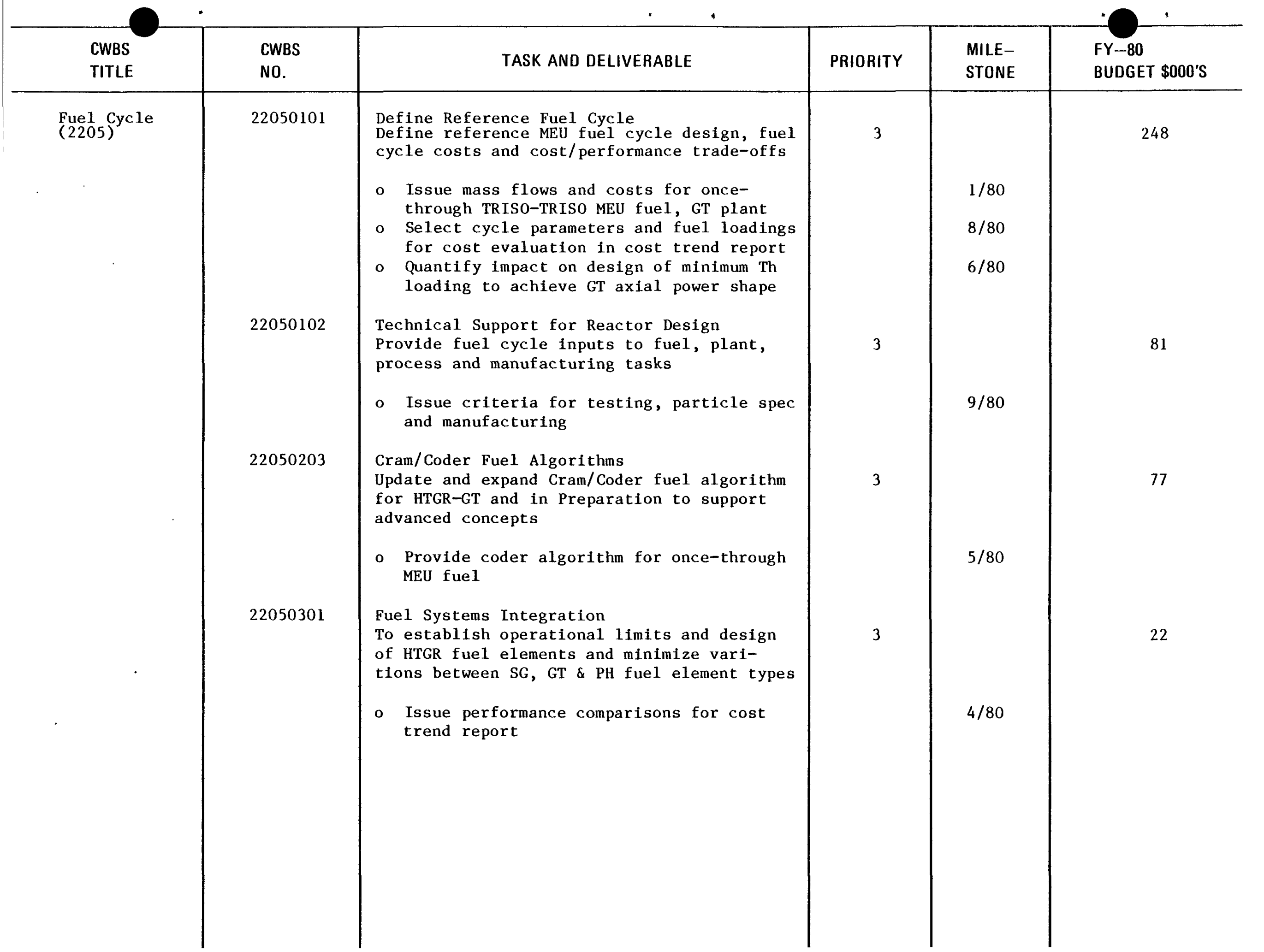




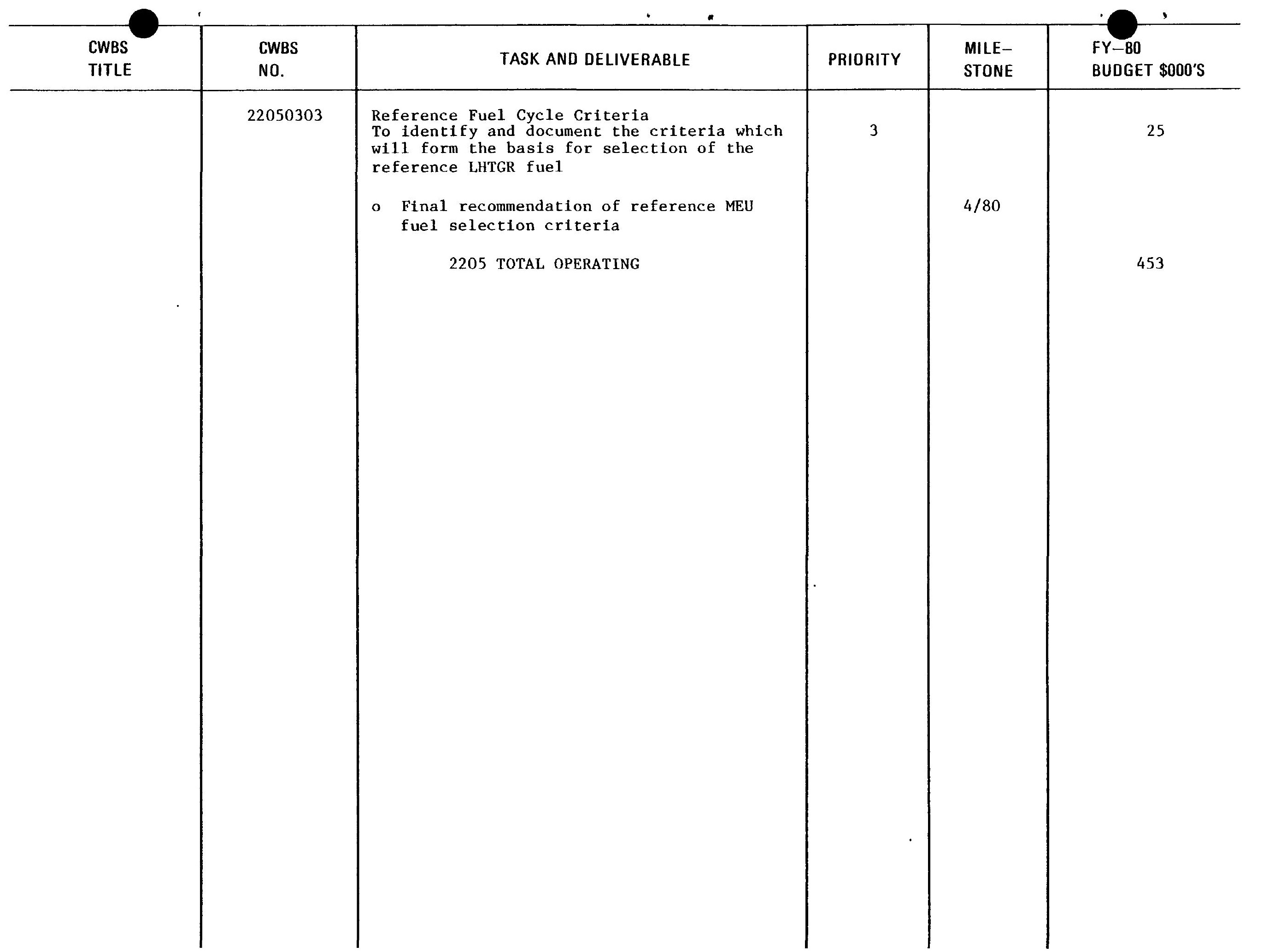




\begin{tabular}{|c|c|c|c|c|c|}
\hline $\begin{array}{l}\text { CWBS } \\
\text { TITLE }\end{array}$ & $\begin{array}{l}\text { CWBS } \\
\text { NO. }\end{array}$ & TASK AND DELIVERABLE & PRIORITY & $\begin{array}{l}\text { MILE- } \\
\text { STONE }\end{array}$ & $\begin{array}{l}\text { FY }-80 \\
\text { BUDGET } \$ 000 ' S\end{array}$ \\
\hline $\begin{array}{l}\text { Fission } \\
\text { Product/ } \\
\text { Coolant } \\
\text { Chemistry } \\
(2206)\end{array}$ & $\begin{array}{l}22060101 \\
22060102 \\
22060103\end{array}$ & $\begin{array}{l}\text { Fission Product Release and Transport } \\
\text { Perform fission product release and trans- } \\
\text { port studies, and provide fission product } \\
\text { data base in support of fission product } \\
\text { design, (a) } \\
\text { o Issue test plan for Cs/Ag release from } \\
\text { MEU fuel particles } \\
\text { Issue status report on Cs/Ag diffusion in } \\
\text { graphite } \\
\text { o Issue status report on fission product } \\
\text { release-from MEU fuel kernels under } \\
\text { normal operating conditions } \\
\text { Fission Product Methods Validation } \\
\text { Validate fission product design methods for } \\
\text { maintenance, containment design and acci- } \\
\text { dent analyses (b) } \\
\text { (a) Work supports proposed PWS FP-2 } \\
\text { (b) Work supports proposed PWS PP-3, CC-1 } \\
\text { o Issue report on Cs release design methods } \\
\text { verifications using IDYLE 03 data } \\
\text { o Issue analyses report on validation with } \\
\text { Dragon plateout data distributions } \\
\text { Fission Product Plateout Studies } \\
\text { Determine plateout characteristics of Cs, } \\
\text { Ag, on turbine materials, for safety, main- } \\
\text { tenance shielding design (c) } \\
\text { o Issue status report on Cs sorption in } \\
\text { turbine alloys } \\
\text { Issue report on Ag sorption tests on tur- } \\
\text { bine alloys }\end{array}$ & $\begin{array}{r}3 \\
-\quad\end{array}$ & $\begin{array}{l}11 / 79 \\
7 / 80 \\
9 / 80\end{array}$ & 151 \\
\hline
\end{tabular}




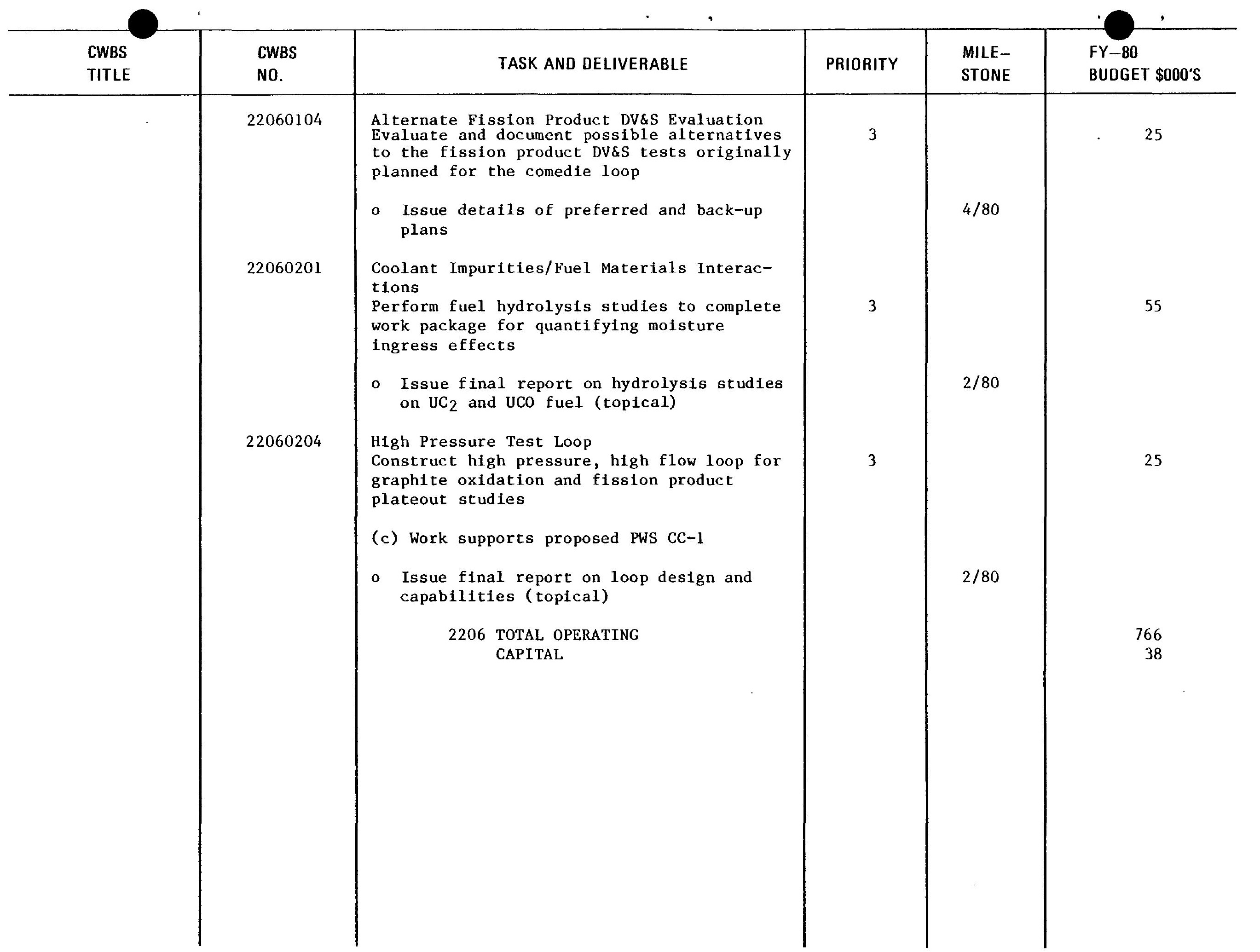




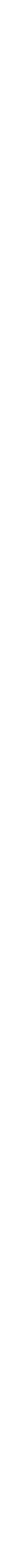




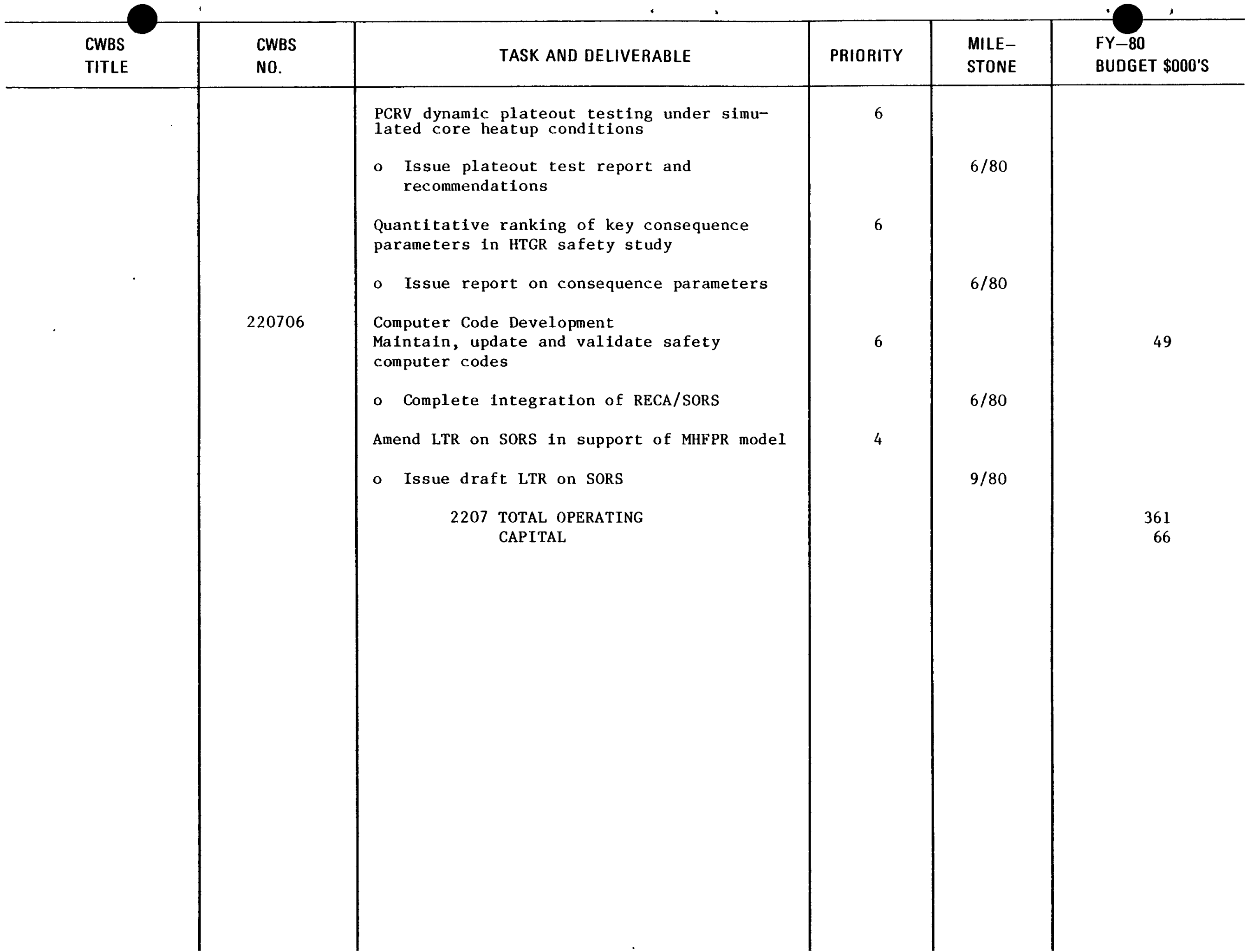




\begin{tabular}{|c|c|c|c|c|c|}
\hline $\begin{array}{l}\text { CWBS } \\
\text { TITLE }\end{array}$ & $\begin{array}{l}\text { CWBS } \\
\text { NO. }\end{array}$ & TASK AND DELIVERABLE & PRIORITY & $\begin{array}{l}\text { MILE- } \\
\text { STONE }\end{array}$ & $\begin{array}{l}\text { FY }-80 \\
\text { BUDGET } \$ 000^{\prime} S\end{array}$ \\
\hline $\begin{array}{l}\text { Materials } \\
\text { Technology } \\
(2208)\end{array}$ & $\begin{array}{l}\text { PWS MC-1 } \\
22081101 \\
\\
\text { PWS MC-5 } \\
22081102 \\
\\
\text { PWS MK-1 } \\
22080401\end{array}$ & $\begin{array}{l}\text { Cooperative Work Under the Umbrella Agreement } \\
\text { Parametric Study of Helium Corrosion } \\
\text { Perform helium impurity corrosion studies, } \\
\text { including parametric testing program. Evalu- } \\
\text { ate carburization and decarburization effects } \\
\text { on structural materials in reactor } \\
\text { environments } \\
\text { o Issue report on initial } \mathrm{CH}_{4} \text { series of } \\
\text { parametric helium impurity corrosion } \\
\text { tests } \\
\text { Issue report on effects of helium impurity } \\
\text { levels on carburization kinetics of alloys } \\
\text { Issue report on results of } 900^{\circ} \mathrm{C} \text { variable } \\
\text { co parametric helium impurity corrosion } \\
\text { tests } \\
\text { Surface Condition Effects on Helium Corrosion } \\
\text { Perform studies to determine effects of prior } \\
\text { condition of materials surfaces upon carbur- } \\
\text { ization and similar helium impurity corros- } \\
\text { Ion actions } \\
\text { o Topical report on Run } 2 \text { exposure } \\
\text { Effects of TGR Helium on Ceramics } \\
\text { Perform tests and evaluations to determine } \\
\text { effects of exposure to gas reactor helium } \\
\text { impurities upon the characteristics of } \\
\text { ceramics materials } \\
\text { o Topical report describing } 2000 \text { hour } \\
\text { exposure of refracturies in GT helium } \\
\text { 2208 ToTAL APPRoved PWs TASKS }\end{array}$ & 3 & $\begin{array}{l}11 / 79 \\
3 / 80 \\
6 / 80\end{array}$ & 98 \\
\hline
\end{tabular}




\begin{tabular}{|c|c|c|c|c|c|}
\hline $\begin{array}{l}\text { CWBS } \\
\text { TITLE }\end{array}$ & $\begin{array}{l}\text { CWBS } \\
\text { NO. }\end{array}$ & TASK AND DELIVERABLE & PRIORITY & $\begin{array}{l}\text { MILE- } \\
\text { STONE }\end{array}$ & $\begin{array}{l}\text { FY-80 } \\
\text { BUDGET } \$ 000 \text { 'S }\end{array}$ \\
\hline & $\begin{array}{l}220801 \\
\\
220803 \\
220804\end{array}$ & $\begin{array}{l}\text { Creep-Fatigue Studies } \\
\text { Perform creep-fatigue tests on base line } \\
\text { alloys; develop fatigue and creep-fatigue } \\
\text { test methods for helium environments; } \\
\text { determine effects of aging and cold working } \\
\text { on creep-fatigue properties of base line } \\
\text { alloys; determine effects of carburization } \\
\text { on low cycle fatigue and creep-fatigue } \\
\text { behavior } \\
\text { o Issue report on results of low cycle fa- } \\
\text { tigue testing of inconel } 617 \\
\text { Issue report on effect of helium environ- } \\
\text { ment on creep fatigue behavior of a1loy } \\
800 H \\
\text { Thermal Aging Studies } \\
\text { Perform tests and analyses to determine } \\
\text { effects of aging on materials behavior } \\
\text { o Issue topical report on the microstruc- } \\
\text { ture and mechanical properties of Incone1 } \\
617 \text {, Alloy } 800 \mathrm{H} \text { and Hastelloy } \mathrm{X} \text { after } \\
\text { aging for } 20,000 \text { hours } \\
\text { Ceramics } \\
\text { Perform core support pad ceramics studies; } \\
\text { perform cover block ceramics studies; per- } \\
\text { form tests and evaluations on ceramics for } \\
\text { ducts and cover plates; perform tests on } \\
\text { fibrous insulation } \\
\text { o Topical report describing flexure strength } \\
\text { test results of al1 candidates } 20^{\circ}-1200^{\circ} \mathrm{C} \\
\text { Topical report describing } 5000 \text { hour } \\
\text { resiliency test results of Saffil Al } 2_{3}{ }_{3} \\
\text { Saffil } \mathrm{Zr0} 2 \text { and graphite felts at } 900^{\circ} \mathrm{C} \\
\text { Draft PWs MK-2 Carbon-Carbon Composite } \\
\text { Materials Studies }\end{array}$ & 3 & $\begin{array}{l}4 / 80 \\
9 / 80 \\
9 / 80\end{array}$ & 235 \\
\hline
\end{tabular}




\begin{tabular}{|c|c|c|c|c|c|}
\hline $\begin{array}{l}\text { CWBS } \\
\text { TITLE }\end{array}$ & $\begin{array}{l}\text { CWBS } \\
\text { NO. }\end{array}$ & TASK AND DELIVERABLE & PRIORITY & $\begin{array}{l}\text { MILE- } \\
\text { STONE }\end{array}$ & $\begin{array}{l}\text { FY }-80 \\
\text { BUDGET } \$ 000^{\prime} S\end{array}$ \\
\hline & $\begin{array}{l}220806 \\
22080601 \\
220807 \\
207\end{array}$ & $\begin{array}{l}\text { Wear and Surface Technology } \\
\text { Perform tests and evaluations of wear } \\
\text { protection coatings in reactor environments; } \\
\text { conduct self-adhesion tests; perform surface } \\
\text { evaporation studies; perform studies of } \\
\text { carburization resistant surfaces } \\
\text { o Topical report describing potential wear } \\
\text { protection coatings, test techniques and } \\
\text { evaluation criteria } \\
\text { Complete } 1000 \text { and } 3000 \text { hour surface } \\
\text { evaporation tests and report the results } \\
\text { Draft Pws MF-1 coating Evaluation for Wear } \\
\text { Protection } \\
\text { Carburization-Resistant claddings and } \\
\text { Coatings } \\
\text { Evaluate commercial and experimental clad- } \\
\text { dings and coatings for prevention of carbur- } \\
\text { ization due to reaction of alloys with pri- } \\
\text { mary coolant impurities } \\
\text { o Issue report on evaluation of protection } \\
\text { carburization by cladding } \\
\text { o Issue report on tests and status of plans } \\
\text { for oxidation-based coatings } \\
\text { Weld Properties } \\
\text { Perform weld properties tests on baseline } \\
\text { alloys; evaluate jointing methods for } \\
\text { advanced alternate alloys } \\
\text { o Preliminary report on weldability of can- } \\
\text { didate materials and properties } \\
\text { o Status report on the joining processes } \\
\text { and evaluation of weldments made in both } \\
\text { wrought and cast a1loys }\end{array}$ & 3 & $\begin{array}{l}3 / 80 \\
9 / 80\end{array}$ & 230 \\
\hline
\end{tabular}




\begin{tabular}{|c|c|c|c|c|c|}
\hline $\begin{array}{l}\text { CWBS } \\
\text { TITLE }\end{array}$ & $\begin{array}{l}\text { CWBS } \\
\text { NO. }\end{array}$ & TASK AND DELIVERABLE & PRIORITY & $\begin{array}{l}\text { MILE- } \\
\text { STONE }\end{array}$ & $\begin{array}{l}\text { FY }-80 \\
\text { BUDGET } \$ 000 \text { 'S }\end{array}$ \\
\hline & $\begin{array}{l}220808 \\
220809 \\
22081100\end{array}$ & $\begin{array}{l}\text { Materials Reliability and Applications } \\
\text { Perform tests and provide design properties } \\
\text { data for baseline alloys for GCRs; provide } \\
\text { generic component design review and support; } \\
\text { study effects of decontamination methods on } \\
\text { metals; determine feasibility of using cast } \\
\text { thermal barrier cover plates; perform } \\
\text { studies on applicability of materials in } \\
\text { alternate GT and VHTR systems } \\
\text { o Issue report updating design correlations } \\
\text { on Hastelloy X, Inconel } 617 \text {, 800H, and } \\
\text { IN loo, to include preliminary cyclic } \\
\text { behavior conditions } \\
\text { Issue report on IHX materials design/life } \\
\text { limits } \\
\text { orovide preliminary report on temperature } \\
\text { design limits for thermal barrier. } \\
\text { Creep Rupture Behavior in HTGR Helium } \\
\text { Determine the effects of HTGR Helium envir- } \\
\text { onment on the creep-rupture properties of } \\
\text { candidate alloys for use in reactor } \\
\text { components } \\
\text { o Issue report on short-term creep-rupture } \\
\text { properties of carburized Alloys } 800 \mathrm{H} \text { and } \\
\text { Hastelloy X } \\
\text { Issue report on creep-rupture behavior of } \\
\text { Alloy 800H and Hastelloy X in simulated } \\
\text { GCR helium } \\
\text { HTGR Helium Compatibility Studies } \\
\text { Study of the effects of long term exposure } \\
\text { in a controlled impurity helium environment } \\
\text { on the corrosion and mechanical behavior of } \\
\text { candidate advanced HTGR structural materials }\end{array}$ & 3 & $\begin{array}{l}9 / 80 \\
12 / 79 \\
8 / 80\end{array}$ & 148 \\
\hline
\end{tabular}




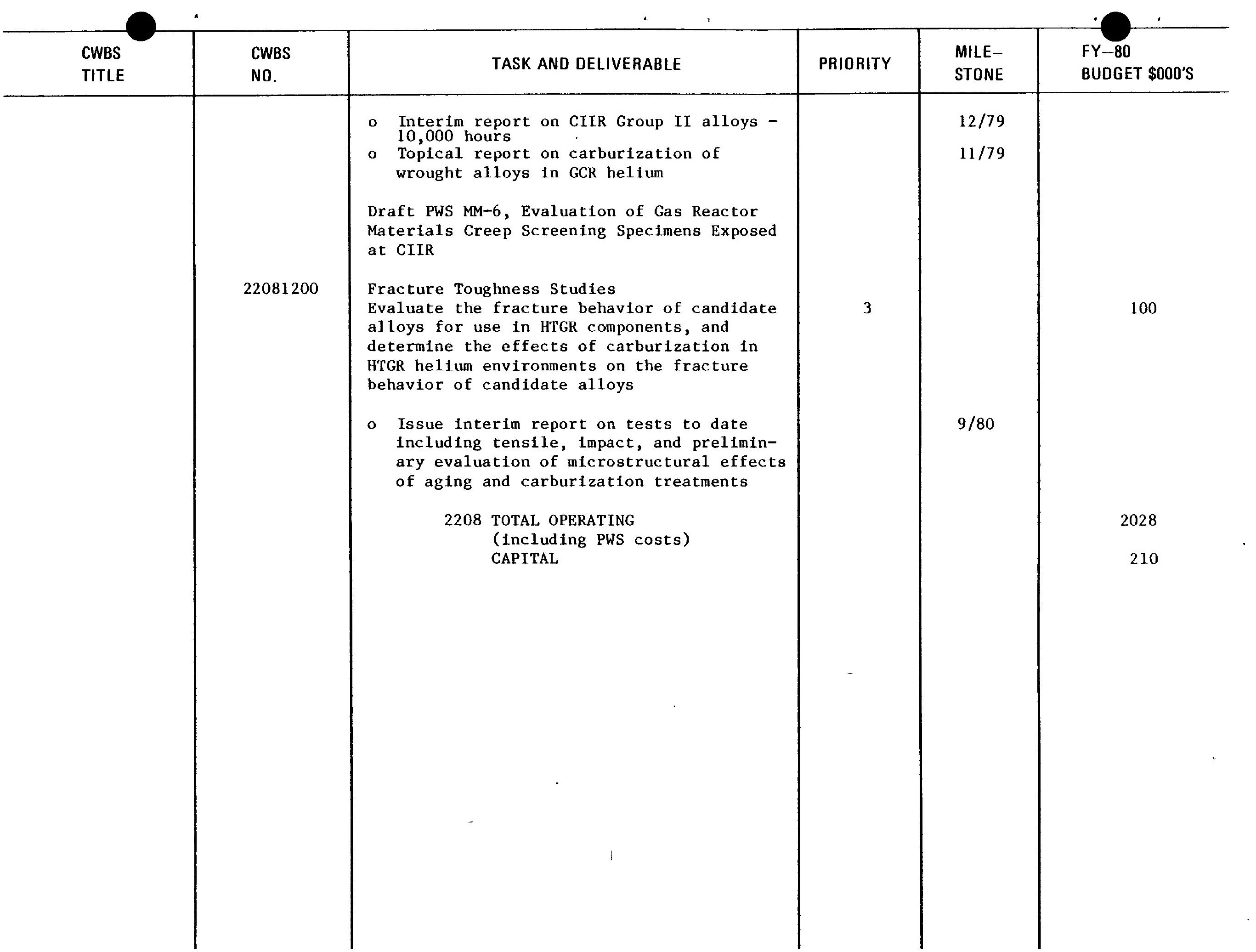




\begin{tabular}{|c|c|c|c|c|c|}
\hline $\begin{array}{l}\text { CWBS } \\
\text { TITLE }\end{array}$ & $\begin{array}{l}\text { CWBS } \\
\text { NO. }\end{array}$ & TASK AND DELIVERABLE & PRIORITY & $\begin{array}{l}\text { MILE- } \\
\text { STONE }\end{array}$ & $\begin{array}{l}\text { FY }-80 \\
\text { BUDGET } \$ 000 ' S\end{array}$ \\
\hline $\begin{array}{l}\text { Structural } \\
\text { Technology } \\
\text { (2209) }\end{array}$ & $\begin{array}{l}22090100 \\
22090300 \\
22090401 \\
22090402\end{array}$ & $\begin{array}{l}\text { PCRV Technology } \\
\text { Provide analytical evaluation of long-term } \\
\text { structural behavior of off-set core type of } \\
\text { PCRV } \\
\text { o Issue report on preliminary results on } \\
\text { evaluation of long-term behavior } \\
\text { Liner Technology } \\
\text { Provide information identified by NRC to } \\
\text { resolve design bases for penetration and } \\
\text { closure failure and develop liner design } \\
\text { criteria for fracture toughness } \\
\text { Fuel Element Graphite Design Technology } \\
\text { Develop and verify design technology and } \\
\text { design criteria for fuel element and re- } \\
\text { placeable reflector graphite } \\
\text { o Issue interim graphite design criteria } \\
\text { o Issue report on non-linear multiaxial, } \\
\text { stress-strain model extended to } \\
\text { irradiation } \\
\text { o Issue load superposition criteria } \\
\text { Core Support Graphite Design Technology } \\
\text { Develop and verify design technology and } \\
\text { design criteria for core support and } \\
\text { permanent reflector graphites } \\
\text { o Issue interim design criteria } \\
\text { o Issue revised draft of ASME Code Sub- } \\
\text { section CE with Committee comments } \\
\text { resolved } \\
\text { o Issue report on discussions of design } \\
\text { criteria with NRC }\end{array}$ & 6 & 4/80 & 20 \\
\hline
\end{tabular}




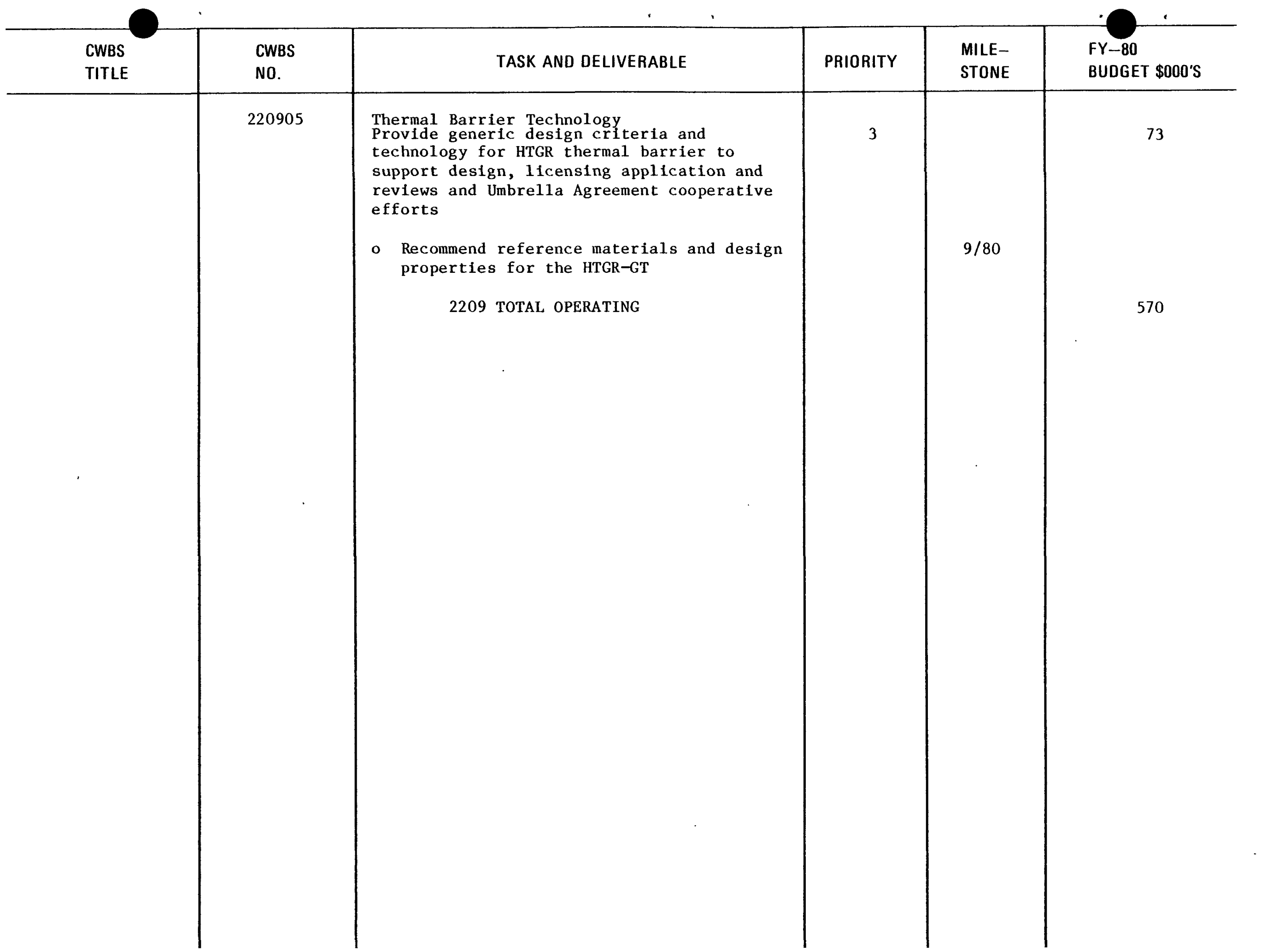




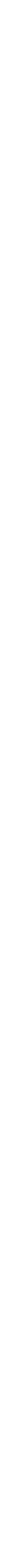




\begin{tabular}{|c|c|c|c|c|c|}
\hline $\begin{array}{l}\text { CWBS } \\
\text { TITLE }\end{array}$ & $\begin{array}{l}\text { CWBS } \\
\text { NO. }\end{array}$ & TASK AND DELIVERABLE & PRIORITY & $\begin{array}{l}\text { MILE- } \\
\text { STONE }\end{array}$ & $\begin{array}{l}\text { FY }-80 \\
\text { BUDGET } \$ 000^{\prime} S\end{array}$ \\
\hline $\begin{array}{l}\text { Heat Exchanger } \\
\text { Base Technology } \\
(2214)\end{array}$ & $\begin{array}{l}221401 \\
22140101 \\
221403 \\
22140301 \\
221405\end{array}$ & $\begin{array}{l}\text { Finned Tube Heat Transfer and Pressure Drop } \\
\text { Test } \\
\text { Characterize finned tube heat transfer and } \\
\text { pressure drop parameters to allow confirm- } \\
\text { ation of GT-HTGR precooler cavity diameter } \\
\text { and overall pressure drop } \\
\text { o Complete model fabrication } \\
\text { o Continue test program } \\
\text { Stayed Tubesheet Recuperator Air Flow Test } \\
\text { o Issue summary test report } \\
\text { Feasibility of Finned Tube ISI } \\
\text { Demonstrate feasible methods of inservice } \\
\text { inspection of finned tubing to meet current } \\
\text { section XI ASME B\&PV code requirements } \\
\text { o Issue test specification } \\
\text { Tube Fretting Wear Test Rig } \\
\text { Complete test rig set-up in preparation } \\
\text { for scoping tests } \\
\text { o Complete check-out report } \\
\text { Materials and Subcontract Test Support } \\
\text { Provide engineering support to materials and } \\
\text { subcontractor tests } \\
\text { o Complete support for testing } \\
\text { 2214 ToTAL opERATING }\end{array}$ & 2 & $\begin{array}{l}6 / 80 \\
9 / 80 \\
4 / 80\end{array}$ & 40 \\
\hline
\end{tabular}




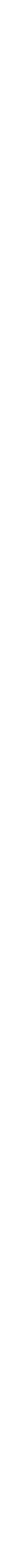




\begin{tabular}{|c|c|c|c|c|c|}
\hline $\begin{array}{l}\text { CWBS } \\
\text { TITLE }\end{array}$ & $\begin{array}{l}\text { CWBS } \\
\text { NO. }\end{array}$ & TASK AND DELIVERABLE & PRIORITY & $\begin{array}{l}\text { MILE- } \\
\text { STONE }\end{array}$ & $\begin{array}{l}\text { FY-80 } \\
\text { BUDGET } \$ 000 ' S\end{array}$ \\
\hline $\begin{array}{l}\text { Reactor Core } \\
\text { Design (2318) }\end{array}$ & $\begin{array}{l}23180101 \\
23180102 \\
23180103\end{array}$ & $\begin{array}{l}\text { Core Generic Nuclear Design } \\
\text { Complete optimization study and design basic } \\
\text { nuclear parameters for HTGR conceptual } \\
\text { design freeze. Complete preliminary } \\
\text { transient and safety analysis } \\
\text { o Complete core design input for cost } \\
\text { estimate } \\
\text { o Provide preliminary core transient data } \\
\text { and safety analysis } \\
\text { o Complete optimization studies for core } \\
\text { conceptual design freeze } \\
\text { Generic Core Performance Analysis } \\
\text { Complete core thermal/flow/performance } \\
\text { part of core conceptual design study and } \\
\text { define basic parameters for LHTGR conceptual } \\
\text { design freeze } \\
\text { o Complete preliminary U0 } 2 \text { fuel performance } \\
\text { analysis } \\
\text { Issue report on } 800 \text { MW GT core } \\
\text { performance analysis } \\
\text { Generic Core Fission Product Analysis } \\
\text { Complete fission product analyses for core } \\
\text { optimization study and define basic param- } \\
\text { eters for LHTGR conceptual design freeze } \\
\text { o Complete preliminary fission product } \\
\text { release from } 800 \text { MW(e) GT core } \\
\text { o Issue radionuclide design criteria for } \\
\text { conceptual design freeze }\end{array}$ & 3 & $\begin{array}{l}5 / 80 \\
8 / 80 \\
9 / 80\end{array}$ & 166 \\
\hline
\end{tabular}




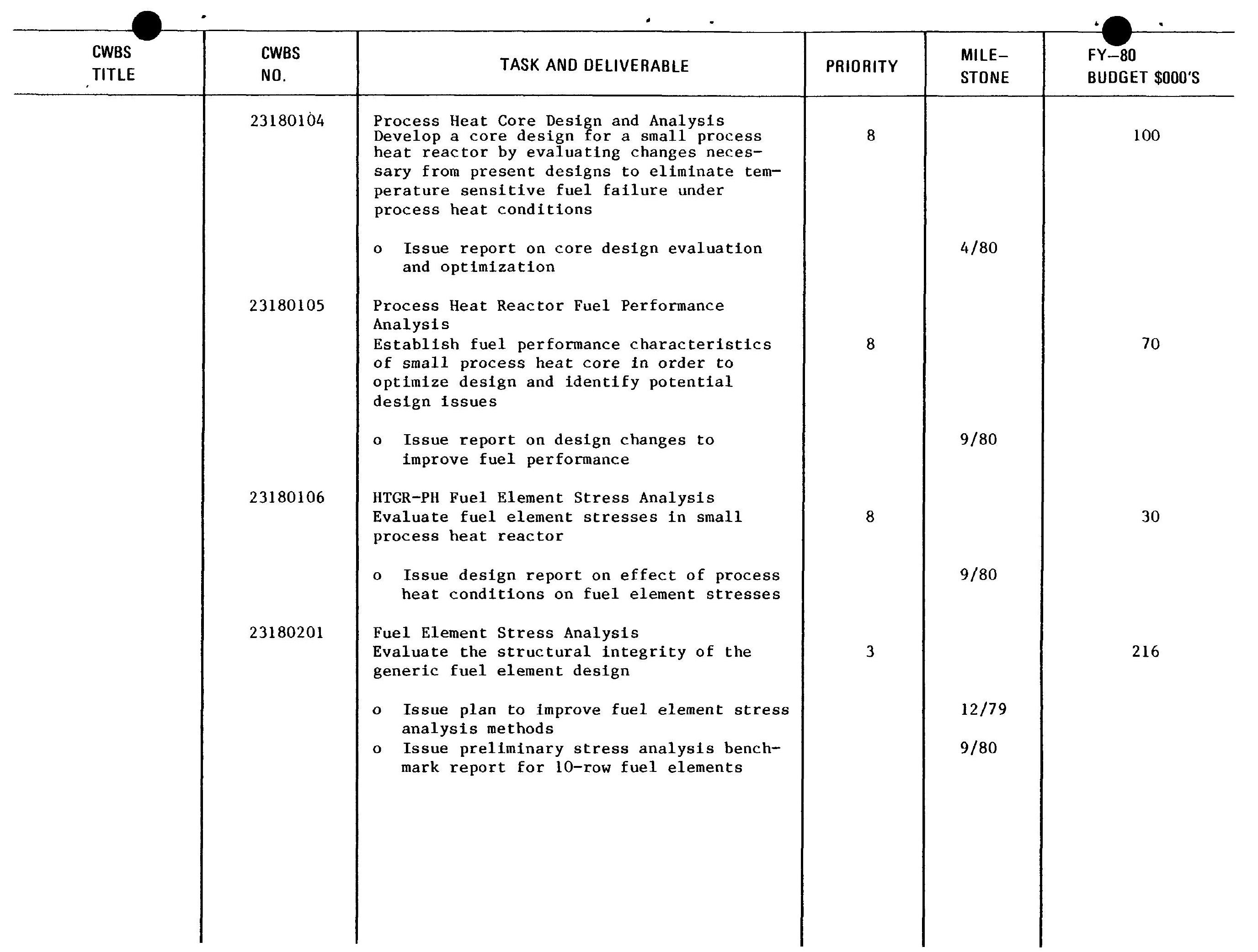




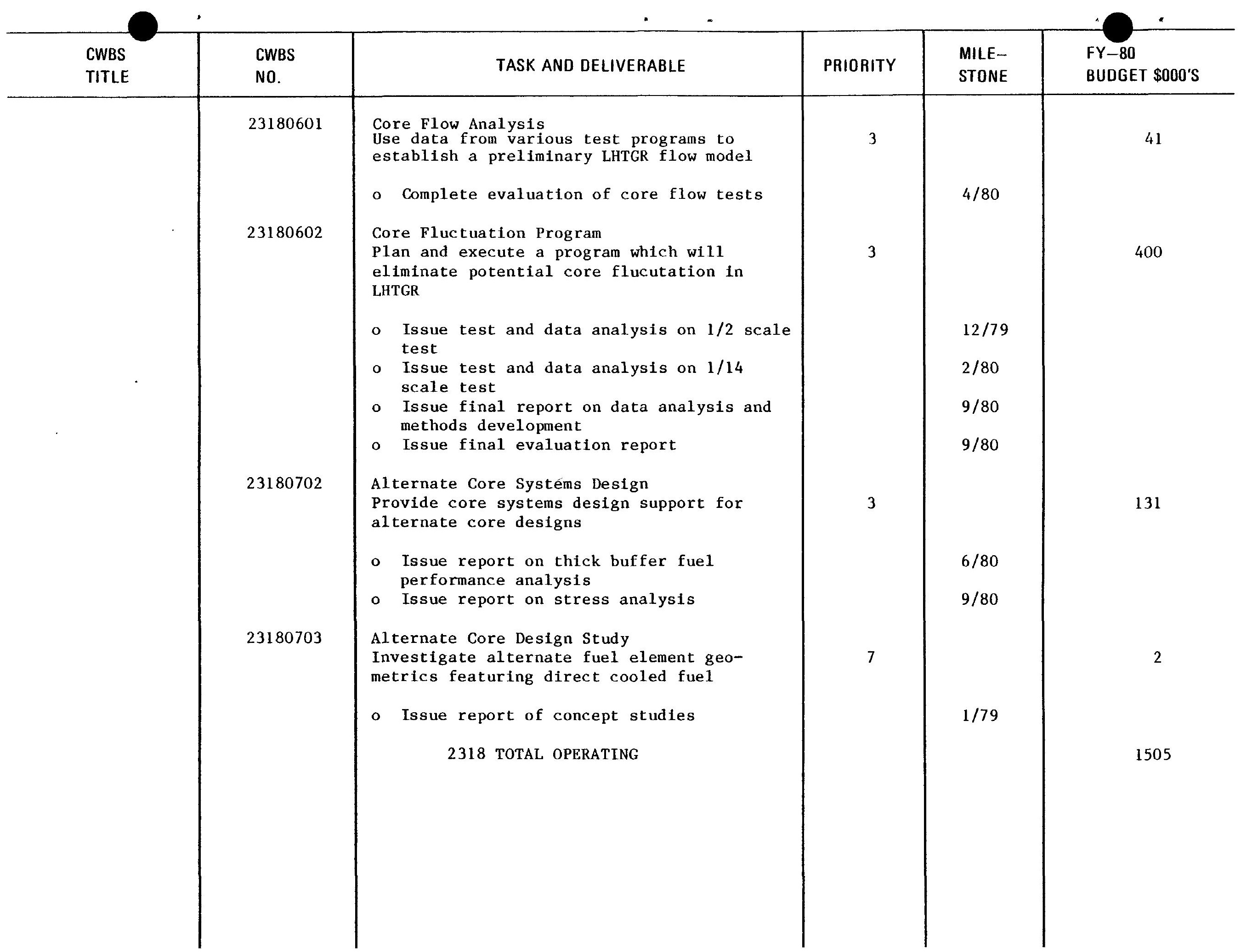




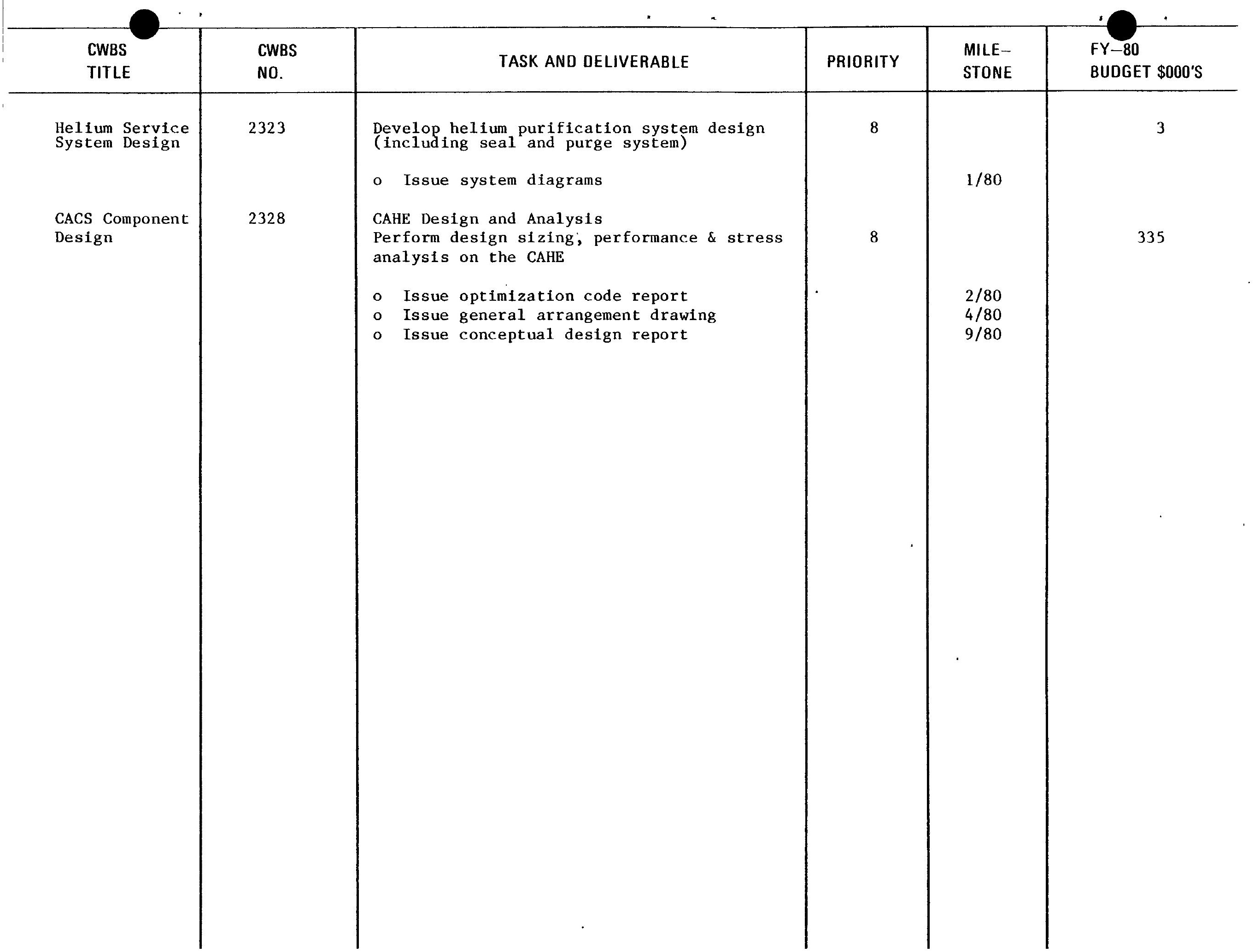




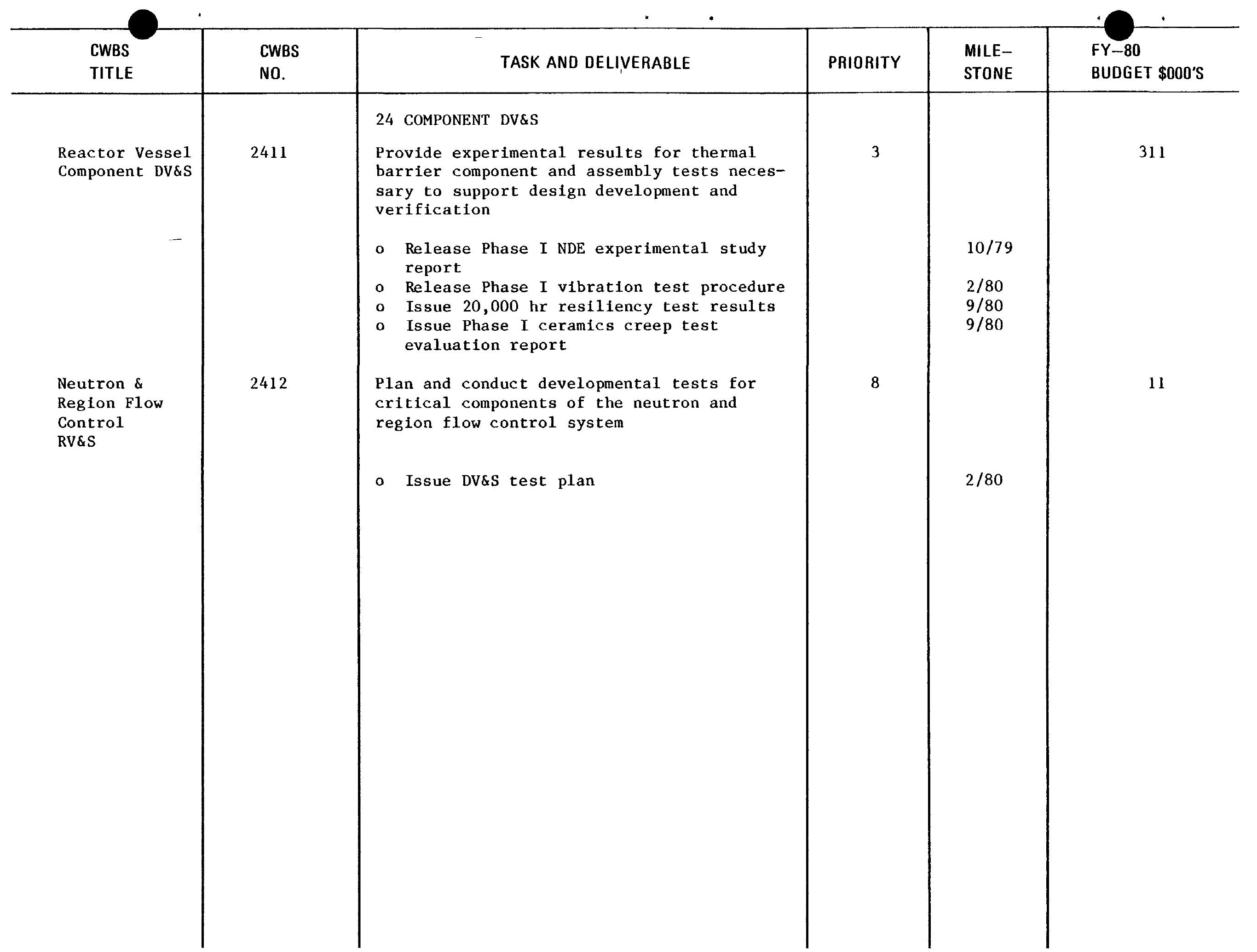




\begin{tabular}{|c|c|c|c|c|c|}
\hline $\begin{array}{l}\text { CWBS } \\
\text { TITLE }\end{array}$ & $\begin{array}{l}\text { CWBS } \\
\text { NO. }\end{array}$ & TASK AND DELIVERABLE & PRIORITY & $\begin{array}{l}\text { MILE- } \\
\text { STONE }\end{array}$ & $\begin{array}{l}\text { FY-80 } \\
\text { BUDGET } \$ 000 \text { 'S }\end{array}$ \\
\hline $\begin{array}{l}\text { Reactor Core } \\
\text { DV \&S } \\
(2418)\end{array}$ & $\begin{array}{l}241801 \\
241802\end{array}$ & $\begin{array}{l}\text { Fuel Element Design Verification } \\
\text { Verify the structural design of fuel ele- } \\
\text { ments and verify application of methods by } \\
\text { comparison of analysis with experiments } \\
\text { performed on components } \\
\text { o Issue report on RWG-2 experiment } \\
\text { o Issue report on RWG-1 experiment } \\
\text { o Issue report on thermal \& stress analysis } \\
\text { of RWG-2 experiment } \\
\text { Core Fluctuation Program } \\
\text { Evaluate data from experimental tests, plan } \\
\text { and execute program to assure against large } \\
\text { HTGR core fluctuations } \\
\text { o List of selected solutions for further } \\
\text { evaluation } \\
\text { o Final report on data analysis, addition } \\
\text { tests and model development } \\
\text { o Final evaluation report on design } \\
\text { solutions } \\
\text { 24l8 TOTAL OPERATING }\end{array}$ & 2 & $\begin{array}{l}3 / 80 \\
9 / 80 \\
9 / 80\end{array}$ & 400 \\
\hline
\end{tabular}




\begin{tabular}{|c|c|c|c|c|c|}
\hline $\begin{array}{l}\text { CWBS } \\
\text { TITLE }\end{array}$ & $\begin{array}{l}\text { CWBS } \\
\text { NO. }\end{array}$ & TASK AND DELIVERABLE & PRIORITY & $\begin{array}{l}\text { MILE- } \\
\text { STONE }\end{array}$ & $\begin{array}{l}\text { FY }-80 \\
\text { BUDGET \$000'S }\end{array}$ \\
\hline & $\begin{array}{r}2804 \\
2805\end{array}$ & $\begin{array}{l}28 \text { PEBBLE BED EVALUATION } \\
\text { Program Management Pebble Bed Evaluation } \\
\text { Provide program management and support to } \\
\text { plan, implement and control the program } \\
\text { o Issue draft evaluation report to ORNL } \\
\text { Core Design } \\
\text { Establish core parameters and perform } \\
\text { evaluation } \\
\text { o Provide power/fluence for } 3000 \text { MW(t) } \\
\text { PBR for HEU and MEU fuel } \\
\text { o Provide core fission product release } \\
\text { evaluation } \\
\text { Provide final report on evaluation } \\
\text { of FRG core performance methods } \\
\text { Pebble Bed Reactor Internals Design } \\
\text { Evaluate Pebble Bed reactor internals } \\
\text { structure and components } \\
\text { o Issue input to evaluation report } \\
\text { PBR Availability and Safety Evaluation } \\
\text { Assess availability and safety aspects of } \\
\text { primary circuit fission product inventory } \\
\text { o Issue report on F.P. inventory and core } \\
\text { temperature effects on safety evaluation } \\
\text { Pebble Bed Reactor Fuel Reprocessing } \\
\text { Evaluation } \\
\text { o Issue draft summary report } \\
\quad 28 \text { ToTAL oPERATING }\end{array}$ & 7 & $\begin{array}{l}2 / 80 \\
3 / 80 \\
9 / 80\end{array}$ & $\begin{array}{r}18 \\
518\end{array}$ \\
\hline
\end{tabular}




\begin{tabular}{|c|c|c|c|c|c|}
\hline $\begin{array}{l}\text { CWBS } \\
\text { TITLE }\end{array}$ & $\begin{array}{l}\text { CWBS } \\
\text { NO. }\end{array}$ & TASK AND DELIVERABLE & PRIORITY & $\begin{array}{l}\text { MILE- } \\
\text { STONE }\end{array}$ & $\begin{array}{l}\text { FY }-80 \\
\text { BUDGET } \$ 000 \text { 'S }\end{array}$ \\
\hline & $\begin{array}{l}31050001 \\
31070001 \\
31080001 \\
31090001 \\
31090100\end{array}$ & $\begin{array}{l}\text { Fresh Fuel Process Development } \\
\text { Document and close-out GA/CEA fresh fuel } \\
\text { process development program } \\
\text { o Issue status report } \\
\text { Heat Exchanger Technology } \\
\text { Analyze and document data from GA/CEA co- } \\
\text { operative heat exchanger R\&D program } \\
\text { o Issue final status report } \\
\text { Materials } \\
\text { Analyze and document data from CEA/GA } \\
\text { materials program } \\
\text { o Issue final report } \\
\text { Fuel Development and Engineering } \\
\text { Perform technical analysis and documentation } \\
\text { of GA fuel performance data from GA/CEA } \\
\text { capsules GF-1, } 2 \text {, } 3 \text {, } 4 \\
\text { o Issue final report on GF-1, } 2 \text {, } 3 \\
\text { o Issue final report on GF-4 } \\
\text { Capsule GF-6, } 7 \text {, Support } \\
\text { Return GA particle samples from Capsule } \\
\text { GF-6 and } 7 \text { to GA for future PIE } \\
\text { (supports PWS FD-9) } \\
\text { o Issue visual examination report }\end{array}$ & 3 & $\begin{array}{l}7 / 80 \\
9 / 80\end{array}$ & 36 \\
\hline
\end{tabular}




\begin{tabular}{|c|c|c|c|c|c|}
\hline $\begin{array}{l}\text { CWBS } \\
\text { TITLE }\end{array}$ & $\begin{array}{l}\text { CWBS } \\
\text { NO. }\end{array}$ & TASK AND DELIVERABLE & PRIORITY & $\begin{array}{l}\text { MILE- } \\
\text { STONE }\end{array}$ & $\begin{array}{l}\text { FY }-80 \\
\text { BUDGET } \$ 000 \text { 'S }\end{array}$ \\
\hline & $\begin{array}{l}31090200 \\
3110\end{array}$ & $\begin{array}{l}\text { Thermal Conductivity Measurement } \\
\text { Apply new Leci device calibration curve to } \\
\text { previously measured fuel rods in order to } \\
\text { obtain more precise calibration curves } \\
\text { Issue final report } \\
\text { Fission Product and Coolant Chemistry } \\
\text { Document and close out GA/CEA fission } \\
\text { product and coolant chemistry program } \\
\text { Issue status report } \\
\quad 31 \text { TOTAL OPERATING }\end{array}$ & 3 & $9 / 80$ & 448 \\
\hline
\end{tabular}




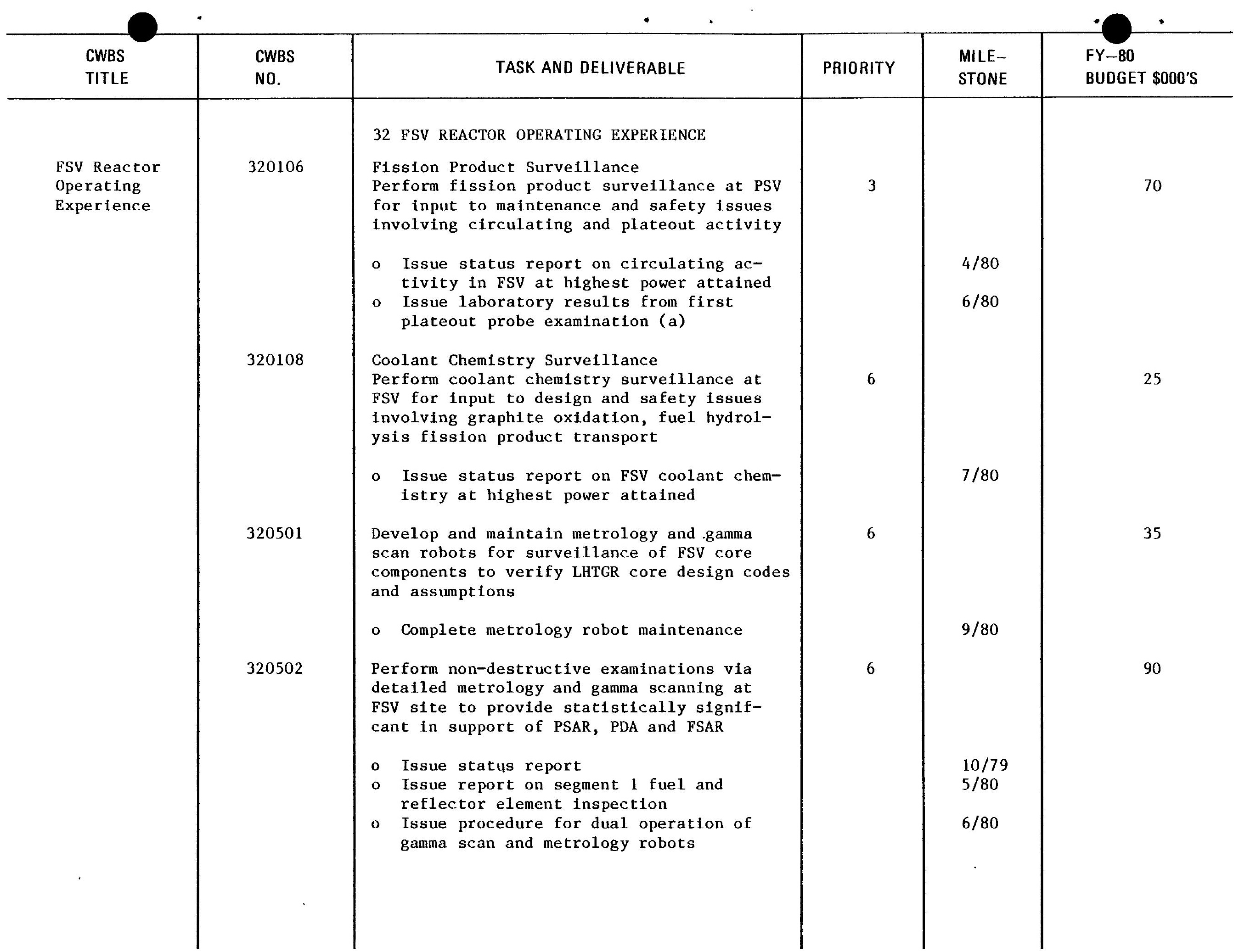




\begin{tabular}{|c|c|c|c|c|c|}
\hline $\begin{array}{l}\text { CWBS } \\
\text { TITLE }\end{array}$ & $\begin{array}{l}\text { CWBS } \\
\text { NO. }\end{array}$ & TASK AND DELIVERABLE & PRIORITY & $\begin{array}{l}\text { MILE- } \\
\text { STONE }\end{array}$ & $\begin{array}{l}\text { FY-80 } \\
\text { BUDGET } \$ 000 \text { 'S }\end{array}$ \\
\hline & $\begin{array}{r}320503 \\
. \\
320600\end{array}$ & $\begin{array}{l}\text { Perform destructive PIEs on fuel elements } \\
\text { reflector elements and control rods to } \\
\text { provide data in supportof PSAR, PDS and FSAR } \\
\text { o Complete fuel rod exam and fission gas } \\
\text { release measurement } \\
\text { o Issue status report } \\
\text { Prepare design guides for HTGRs bases on } \\
\text { FSV construction and operating experience } \\
\text { o Issue mechanical and electrical design } \\
\text { guide } \\
\text { Complete design guide handbook } \\
3200 \text { TOTAL OPERATING }\end{array}$ & 8 & $\begin{array}{l}3 / 80 \\
7 / 80\end{array}$ & 350 \\
\hline
\end{tabular}




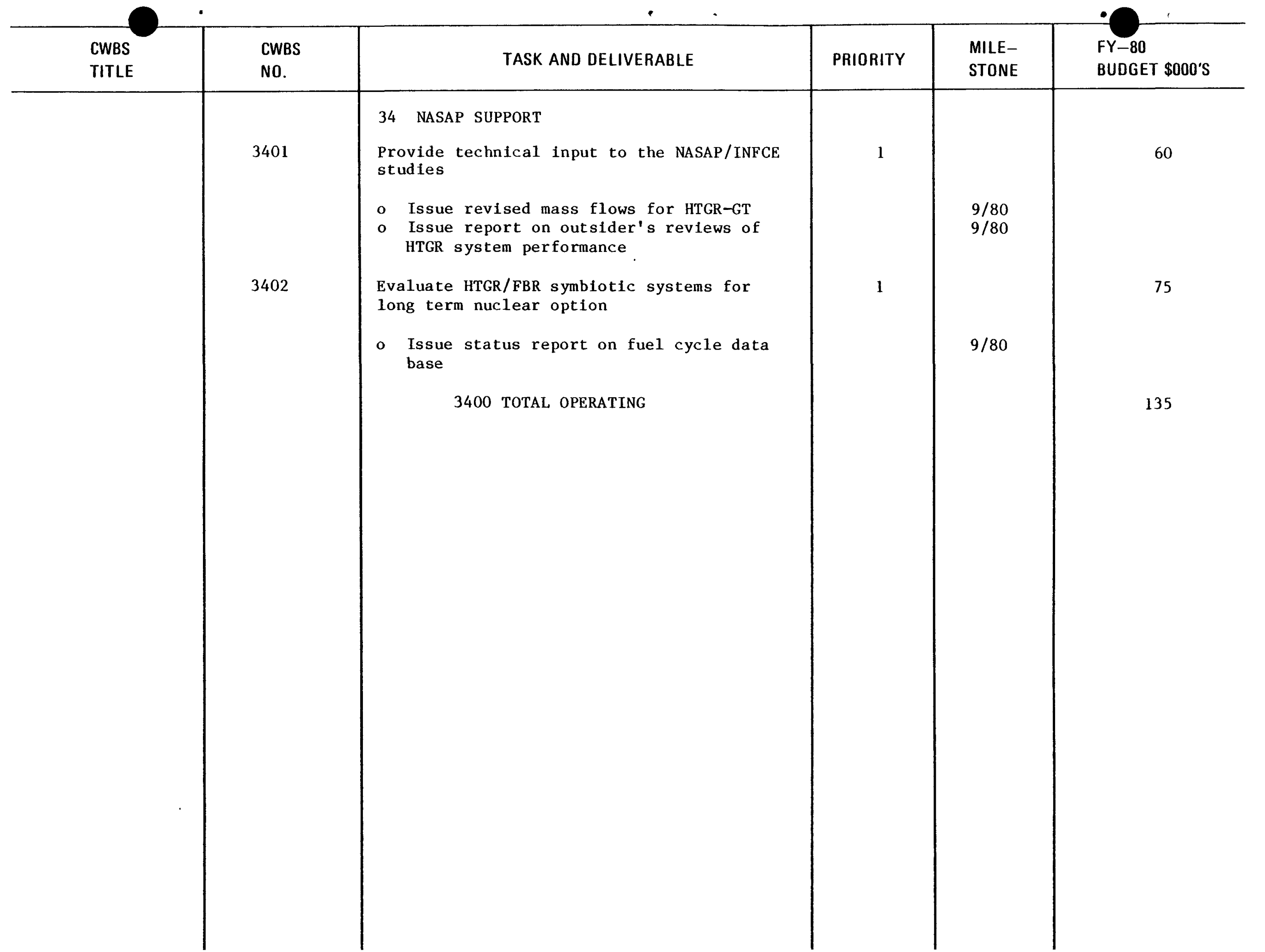




\begin{tabular}{|c|c|c|c|c|c|}
\hline $\begin{array}{l}\text { CWBS } \\
\text { TITLE }\end{array}$ & $\begin{array}{l}\text { CWBS } \\
\text { NO. }\end{array}$ & TASK AND DELIVERABLE & PRIORITY & $\begin{array}{l}\text { MILE- } \\
\text { STONE }\end{array}$ & $\begin{array}{l}\text { FY }-80 \\
\text { BUDGET \$000'S }\end{array}$ \\
\hline $\begin{array}{l}\text { Program Man- } \\
\text { agement and } \\
\text { Support } \\
\text { Activities }\end{array}$ & $\begin{array}{l}9901 \\
9902 \\
9903 \\
9904 \\
9\end{array}$ & $\begin{array}{l}\text { XX99 PROGRAM MANAGEMENT } \\
\text { Program Management } \\
\text { Provide program management support for the } \\
\text { HTGR Generic Technology Program including } \\
\text { planning and scheduling and quality } \\
\text { assurance } \\
\text { o Prepare outline of development plan } \\
\text { o Issue detailed development plan for long } \\
\text { term generic program based on network } \\
\text { logic } \\
\text { Program Planning, Scheduling and Reporting } \\
\text { Provide project planning scheduling and } \\
\text { reporting support to generic program } \\
\text { Quality Assurance } \\
\text { Define and manage the general program } \\
\text { quality assurance program } \\
\text { Licensing } \\
\text { Coordinate preparation of LTRs under pre- } \\
\text { application program and coordinate response } \\
\text { to NRC preview. Prepare status reports on } \\
\text { HTGR-GT DV\&S programs } \\
\text { o Issue general design criteria LTR to GCRA } \\
\text { o Issue time dependent release LTR to GCRA } \\
\text { o Issue accident initiation and progression } \\
\text { LTR to GCRA } \\
\text { o Issue seismic methods verification LTR } \\
\text { input to GCRA } \\
\text { Systems Integration } \\
\text { Provide systems integration review } \\
\text { of design activities }\end{array}$ & 1 & $\begin{array}{l}11 / 79 \\
1 / 80 \\
6 / 80 \\
9 / 80\end{array}$ & 1043 \\
\hline
\end{tabular}


6.3 FOREIGN TRAVEL PLANS: FY-80

\begin{tabular}{|c|c|c|c|c|}
\hline CWBS & & & Organization & No. \\
\hline No. & Title & Trip/Purpose & To Be Visited & Trips \\
\hline $\begin{array}{l}2201 \\
2202\end{array}$ & $\begin{array}{l}\text { Fuel Process/ } \\
\text { Fuel Development }\end{array}$ & $\begin{array}{l}\text { Develop fuel graphite, } \\
\text { F.P. subprogram plan } \\
\text { Obtain data on the } \\
\text { R2-K-13 Capsule }\end{array}$ & $\begin{array}{l}\text { HOBEG } \\
\text { KFA }\end{array}$ & 2 \\
\hline 2203 & Graphite Development & $\begin{array}{l}\text { Development of Graphite } \\
\text { Design Criteria }\end{array}$ & $\mathrm{KFA}, \mathrm{CEGB}$ & 1 \\
\hline 2206 & $\begin{array}{l}\text { Fission Product \& } \\
\text { Coolant Chemistry }\end{array}$ & $\begin{array}{l}\text { Review of PWS CCl \& FP-3. } \\
\text { Develop co-operative } \\
\text { test plans. }\end{array}$ & KFA & 1 \\
\hline 2207 & Safety \& Reliability & $\begin{array}{l}\text { Information Exchange. } \\
\text { AIPA Review SAI \& } 2\end{array}$ & $\mathrm{KFA}$ & 1 \\
\hline 2208 & Materials Technology & $\begin{array}{l}\text { Discuss PWS Test Results } \\
\& \text { Review Program Status }\end{array}$ & $\begin{array}{l}\text { KFA } \\
\text { HTMP } \\
\text { CIIR }\end{array}$ & 2 \\
\hline 2209 & Structural Technology & $\begin{array}{l}\text { Technical Review of } \\
\text { Project Work Statement } \\
\text { on PCRV, Liner, } \\
\text { Thermal Barrier }\end{array}$ & $\begin{array}{l}\text { KFA } \\
\text { HRB } \\
\text { EIR }\end{array}$ & 1 \\
\hline 2214 & $\begin{array}{l}\text { Heat Exchanger } \\
\text { Technology }\end{array}$ & $\begin{array}{l}\text { Evaluation of Test Models } \\
\& \text { Test Data }\end{array}$ & Sulzer & 1 \\
\hline 2299 & Program Management & $\begin{array}{l}\text { Overall base technology } \\
\text { data exchange, program } \\
\text { development and CEA } \\
\text { Subcontract liaison }\end{array}$ & $\begin{array}{l}\text { KFA } \\
\text { CEA } \\
\text { EIR }\end{array}$ & 1 \\
\hline $\begin{array}{l}2318 / \\
2418\end{array}$ & Core Design & Proteus Experiment & EIR & 1 \\
\hline $28 \times x$ & $\begin{array}{l}\text { Pebble Bed } \\
\text { Evaluation }\end{array}$ & $\begin{array}{l}\text { Review Status of FRG } \\
\text { Design with Emphasis } \\
\text { on Reactor Core and } \\
\text { Internals Structure }\end{array}$ & $\begin{array}{l}\mathrm{HRB}, \mathrm{GHT} \\
\mathrm{KFA}\end{array}$ & 2 \\
\hline $31 x x$ & CEA Liaison & $\begin{array}{l}\text { Relocation from Saclay } \\
\text { of GA Test Liaison } \\
\text { Engineer. CEA test } \\
\text { programs and test data } \\
\text { review. Subcontract } \\
\text { program review } \\
\quad \text { TOTAL TRIPS }\end{array}$ & CEA & 15 \\
\hline
\end{tabular}

